\title{
Taxonomía de onicóforos de Santander, Colombia y termogravimetría, calorimetría de barrido diferencial y espectroscopía infrarroja de la secreción adhesiva (Onychophora: Peripatidae)
}

\author{
Javier H. Jerez-Jaimes ${ }^{1}$ \& M. Catalina Bernal-Pérez ${ }^{2}$ \\ 1. Escuela de Ingeniería Química, Universidad Industrial de Santander, Calle 9 Cra. 27 Bucaramanga, Colombia; \\ jaherjer@uis.edu.co \\ 2. Escuela de Biología, Universidad Industrial de Santander, Calle 9 Cra. 27 Bucaramanga, Colombia; \\ mcbernalperez@yahoo.es
}

Recibido 10-VII-2008. Corregido 20-III-2009. Aceptado 20-IV-2009

\begin{abstract}
Velvet worm taxonomy from Santander, Colombia and thermogravimetry, differential scanning calorimetry and infrared spectroscopy of the adhesive secretion (Onychophora: Peripatidae). Onychophoran worms are terrestrial organisms that have changed relatively little since the mid-Cambrian. We collected Macroperipatus geagy in coffee plantations at "Hacienda El Roble", Santander, Colombia, and here redescribe the species based on 15 individuals. A digital three-dimensional reconstruction of the ventral side of the body indicates that the primary and accessory papillae lack a defined distribution pattern. Diagnostic characters: one main tooth and one accessory tooth in the outer jaw, and one main tooth, one accessory tooth and seven denticles in the internal jaw. Measurements: length $(X=45.66 \mathrm{~mm} ; \mathrm{SD}=26.10)$, weight $(X=0.95 \mathrm{~g}$; $\mathrm{SD}=2.21)$ and number of lobopods $(\mathrm{X}=28.13$ pairs; $\mathrm{SD}=1.30)$. We present a taxonomic key for six species of Macroperipatus. The liquid adhesive secretion lost $60 \%$ of the initial weight at $70{ }^{\circ} \mathrm{C}$ (solid: $13 \%$ close to 90 $\left.{ }^{\circ} \mathrm{C}\right)$. The stability phase of the liquid secretion was within $90-280{ }^{\circ} \mathrm{C}\left(100{ }^{\circ} \mathrm{C}-205^{\circ} \mathrm{C}\right.$ in the solid secretion). The starting degradation temperature of the sample was $355^{\circ} \mathrm{C}$. Heat flow changes in the solid and liquid secretions were confirmed by the sample behavior during thermogravimetric analysis. The percentage of $\beta$ sheets calculated by infrared spectrum was $59 \%$. In comparison with Nephila spiders, the onychophoran secretion lost more weight and entered the phase of degradation at lower temperatures. This secondary structure of proteins gives the onychophoran adhesive secretion a tensile strength and extensibility similar to those of the silk produced by spiders for prey-capture. Rev. Biol. Trop. 57 (3): 567-588. Epub 2009 September 30.
\end{abstract}

Key words: velvet worms, Macroperipatus geagy, denticles, inner jaw, beta sheet, infrared spectrum, silk spiders.

Los onicóforos o "gusanos aterciopelados" se denominan así debido a la apariencia aterciopelada que proyectan las miles de papilas sensoriales que conforman la cutícula quitinosa que los cubre (Johnson et al. 1969). Los hábitos crípticos, la actividad nocturna debido al fototropismo negativo (Monge-Nájera et al. 1993, 1996) y la baja densidad poblacional hacen que sean raramente observados y por consiguiente difícilmente estudiados (Bouvier 1905, Lavallard 1975, Monge-Nájera y Hou 1999). Estos invertebrados están restringidos a microhabitats húmedos y oscuros, principalmente bajo la hojarasca, dentro de microcavernas del suelo, bajo rocas y en galerías dentro de troncos en descomposición, cerca de algún cauce debido a su incapacidad para controlar la pérdida de agua (Peck 1975, Monge-Nájera y Alfaro 1995, Gleeson 1996, Monge-Nájera y Hou 1999). Son depredadores nocturnos y debido a su lenta movilidad atrapan a sus presas (otros pequeños invertebrados como termitas, isópodos, lombrices y larvas de escarabajos) (Rupert y Barnes 1996, Monge-Najera 
y Hou 1999, Vasconcellos et al. 2006) expulsando sobre estos una sustancia pegajosa de carácter proteico (Roper 1977) desde un par de extremidades modificadas llamadas papilas orales o glándulas de la seda situadas a ambos lados de la región cefálica (Monge-Nájera et al. 1993, Monge y Morera 1994, Strausfeld et al. 2006). El costo energético de producción de esta secreción es alto, por lo que después de ser expulsada sobre la presa es reingerida junto con ésta (Read y Hughes 1987) siendo también la base de la alimentación de los onicóforos jóvenes después del nacimiento (Morera et al. 1988).

Esta secreción adhesiva implicada en la depredación y defensa es una de las características más distintivas de los miembros del Filo Onychophora. Read en 1985 reportó la presencia de proteínas, carbohidratos, lípidos y agua (85\%) en la secreción adhesiva de Macroperipatus torquatus (Peripatidae). Estudios en cuanto a la composición bioquímica de la secreción adhesiva de Peripatopsis moseleyi (Roper 1977) y Epiperipatus kanangrensis (Benkendorff et al. 1999) (Peripatopsidae) han reportado la presencia de lípidos, carbohidratos, proteína, agua (90\%), nonilfenol como surfactante y glicina, prolina y lisina como los aminoácidos más abundantes en la secreción. Las fibras de la secreción adhesiva de los onicóforos han sido catalogadas como similares a las fibras de seda producidas por las arañas de acuerdo a la alta resistencia a la tensión, extensibilidad y adhesión (Benkendorff et al. 1999, Hayashi y Lewis 1998, Vollrath y Tillinghast 1991).

El Filo Onychophora comprende aproximadamente 200 especies (Mayer 2007) geográficamente distribuidas a lo largo de los continentes que una vez conformaron Gondwana (MongeNájera 1995), desde el centro y sur de América hasta África, incluyendo las partes más húmedas de Australia. Las especies de onicóforos están clasificadas en dos familias, la familia Peripatopsidae (Bouvier 1905) que se distribuye a lo largo de África del Sur, Chile y Australasia, y la familia Peripatidae (Evans 1901a) que ocupa la zona ecuatorial: Antillas, México, América Central, África ecuatorial occidental, región norte de América del Sur y Sudeste de Asia (Bouvier 1905, Monge-Nájera 1995). Las especies de estas familias se caracterizan por la solubilidad (Peripatidae) o no (Peripatopsidae) de los pigmentos del cuerpo en etanol, presencia (Peripatidae) o ausencia (Peripatopsidae) de diastema en las sierras de la mandíbula interna, abertura genital entre el penúltimo (Peripatidae) o último (Peripatopsidae) par de lobópodos y por poseer entre 19 y 43 (Peripatidae), y entre 13 y 29 (Peripatopsidae) pares de lobópodos (Storch y Ruhberg 1993, Monge-Nájera 1995, Mayer 2007).

Peck (1975) recopiló de las familias del filo el listado de especies y géneros de los onicóforos del nuevo mundo, al igual que las áreas de distribución en donde fueron reportadas por primera vez; de la familia Peripatidae fueron reportados para Colombia cuatro géneros: Epiperipatus, Macroperipatus, Oroperipatus y Peripatus, y de estos cuatro géneros, diez especies con distribución en los departamentos de Antioquia, Cundinamarca, Cauca y Magdalena (Cuadro 1). Investigaciones en cuanto a morfología (Brues 1925, Peck 1975, Morera y León 1986, Read 1986, Morera y Monge-Nájera 1990, Monge-Nájera et al. 1993, MongeNájera y Morera 1994, Oliveira y Wieloch 2005, Mayer 2007), ecología y biogeografía (Monge-Nájera 1994 a y b, Monge-Nájera y Alfaro 1995, Monge-Nájera 1995, Mcglynn y Kelley 1999) de estos géneros en Sur América y el Caribe se han realizado con especies de Costa Rica, Brasil y Ecuador en contraste con las especies reportadas para Colombia, de las que actualmente se tiene el mínimo de conocimiento.

De acuerdo con lo anterior y atendiendo a la necesidad de superar dicho vacío, en este trabajo se realiza la redescripción de Macroperipatus geagy (Bouvier 1899b) como la especie habitante de los cafetales de la Mesa de Los Santos y el primer reporte de onicóforos en el departamento de Santander (Colombia). Se realiza la caracterización térmica de la secreción adhesiva de $M$. geagy mediante análisis termogravimétricos (TG) y de calorimetría de barrido diferencial (DSC), analizando la 
CUADRO 1

Géneros y especies de onicóforos (Peripatidae) reportados para Colombia, según Peck (1975)

TABLE 1

Genus and species of onychophorans (Peripatidae) reports for Colombia, according to Peck (1975)

\begin{tabular}{lcccc}
\multicolumn{5}{c}{ Familia Peripatidae } \\
Género & \multicolumn{2}{c}{ Especies } \\
Oroperipatus & O. lankesteri & O. bimbergi & O. multipodes & O. tuberculatus \\
Cunde & Cundinamarca & Antioquia & \\
Epiperipatus & E. edwardsii & E. imthurmi & E. vespucci & \\
& Magdalena & Magdalena & Magdalena & \\
Peripatus & P. juliformis & P. bouvieri & \\
& Occidente de & Cundinamarca & \\
Colombia & & \\
Macroperipatus & M. geayi & & \\
& Magdalena & &
\end{tabular}

historia térmica de la secreción y los cambios de peso y energía en función de la temperatura. Se realiza la caracterización de los componentes químico-estructurales de la secreción adhesiva y se calcula el porcentaje de láminas beta $(\beta)$ de la secreción adhesiva de $M$. geagy mediante espectroscopía de infrarrojos FTIR. Este es el primer estudio termogravimétrico, calorimétrico y de espectroscopía de infrarrojos de la secreción adhesiva de un onicóforo.

\section{MATERIALES Y MÉTODOS}

Área de estudio: El área estudiada pertenece a la hacienda El Roble, Plantación Café Mesa de Los Santos ${ }^{\circledR}$, ubicada a $06^{\circ} 54^{\prime} 38^{\prime \prime}$ latitud Norte, $73^{\circ} 07^{\prime} 45^{\prime \prime}$ longitud Oeste, en la vereda El Carrizal, municipio Los Santos, departamento Santander (Colombia); con una altitud de $1600 \mathrm{~m}$. El hábitat se caracteriza por la presencia de algunos relictos de bosques naturales y por presentar veranos calurosos e inviernos fríos y lluviosos (Peraza et al. 2004), con épocas de alta precipitación entre marzo, mayo, septiembre y noviembre, temperatura de $18{ }^{\circ} \mathrm{C}$ y precipitación promedio anual de 955.8 $\mathrm{mm}$ (datos suministrados por la oficina del
Ministerio del Medio Ambiente de la ciudad de Bucaramanga).

Trabajo de campo: Durante enero y febrero de 2007 se visitaron las zonas rurales del Municipio Los Santos y se realizaron encuestas a los pobladores en cuanto al conocimiento y observación de los onicóforos en la región. De acuerdo con información anecdótica de profesores y estudiantes de la Universidad Industrial de Santander y la información suministrada por los habitantes de la zona, se delimitaron los sitios de muestreo dentro de los cafetales de la hacienda El Roble. Se seleccionaron las áreas de cafetal que se encontraban menos expuestas a la luz solar durante la mayor parte del día ubicadas cerca de las pequeñas corrientes de agua de la hacienda y en las que se observó un mayor número de troncos en descomposición. Cada 20 días y durante cinco meses, se realizaron visitas a las zonas identificadas para la búsqueda de los onicóforos. El método de búsqueda consistió en examinar debajo de la hojarasca del cafetal, debajo de hojas de plátano caídas y dentro de los troncos en descomposición, así como dentro de pequeños montículos de tierra ubicados en el límite del cafetal y el caudal 
de agua. Los onicóforos fueron recolectados manualmente y se transportaron en recipientes plásticos que contenían tierra y trozos de madera húmedos tomados del mismo sitio de muestreo. Los recipientes fueron cerrados herméticamente hasta ser transportados al laboratorio evitando la pérdida de peso de los onicóforos por deshidratación.

Trabajo en el laboratorio: Se realizaron medidas morfométricas (peso, longitud y número de lobópodos) de cada uno de los especímenes recolectados, calculándose la media, desviación estándar y varianza de los datos utilizando el programa para análisis estadístico PAST (Palaeontological Statistics, ver. 1.81). El peso se registró en una balanza analítica y la longitud fue medida sobre una hoja de papel blanca mientras los onicóforos caminaban extendidos en línea recta.

Extracción de la secreción adhesiva: Para la obtención de la secreción adhesiva de los onicóforos para el análisis termogravimétrico, calorimétrico y de espectroscopía de infrarrojos, los onicóforos fueron introducidos en un tubo para micro centrífuga por la región cefálica, estímulo que el onicóforo interpretó como peligro, expulsando la secreción dentro del tubo. Con este procedimiento fue posible almacenar y aislar la muestra adecuadamente, evitando contaminaciones y permitiendo conservar vivo el animal durante un mayor lapso de tiempo debido a que los onicóforos mueren después de haber sufrido un alto nivel de estrés. Las muestras de la secreción adhesiva se almacenaron a temperatura ambiente para posteriormente realizar los análisis térmicos y de infrarrojo. Las muestras sólidas de la secreción se obtuvieron por estimulación del onicóforo para que expulsara la secreción sobre una lámina de vidrio, la cual solidificó a una temperatura de $22{ }^{\circ} \mathrm{C}$.

Identificación taxonómica: Los animales que sobrevivieron al estrés causado por la extracción de la secreción adhesiva y los preservados en alcohol, se observaron en 100, 200 y 400 aumentos a través del video microscopio 3D HIROX 7700 en el laboratorio de biomateriales de la escuela de Ingeniería Metalúrgica de la Universidad Industrial de Santander. La superficie dorsal de la piel de los onicóforos fue reconstruida en un plano $3 \mathrm{D}$ con el fin de observar el patrón de distribución de las papilas dorsales. Se extrajeron las mandíbulas internas y externas de los especímenes y se observaron en 100 aumentos a través del microscopio óptico. Para la extracción de las mandíbulas los especímenes fueron anestesiados con éter para posteriormente realizar una incisión longitudinal en la boca y realizar la extracción. Mediante el uso de la clave taxonómica para onicóforos de Peck (1975), se identificó el género al que pertenecen los especímenes recolectados. La especie se determinó mediante comparaciones de los caracteres morfológicos observados con algunas de las descripciones morfológicas originales realizadas para especies del género Macroperipatus de Costa Rica y Brasil en donde se comparan y describen algunas de las características morfológicas generales de $M$. geagy (ver Bouvier 1907, Brues 1925, Morera y León 1986, Oliveira y Wieloch 2005).

Preservación: Los especímenes fueron preservados en alcohol del $70 \%$. Inicialmente fueron anestesiados con éter y sacrificados en alcohol, sumergidos en formol durante 24 horas y finalmente conservados en alcohol del $70 \%$. Cada espécimen fue depositado en la colección entomológica del Museo de Historia Natural de la Universidad Industrial de Santander en Colombia.

Análisis termogravimétrico (TG): Este tipo de análisis fue implementado con el objetivo de conocer la cantidad de cambio en la masa en función de la temperatura y predecir la estabilidad térmica de la secreción adhesiva de $M$. geagy. Los análisis se realizaron en un termoanalizador $\mathrm{TGA} / 2050$ bajo un régimen de calentamiento dinámico (el aumento de la temperatura es a velocidades constantes). Se realizaron análisis termogravimétricos de muestras de la secreción adhesiva de los 
onicóforos en estado líquido y sólido. De la muestra de secreción adhesiva en estado líquido se utilizaron $5.79 \mathrm{mg}$ y $0.19 \mathrm{mg}$ de la muestra en estado sólido. La velocidad de calentamiento o rampa de temperatura fue de $10{ }^{\circ} \mathrm{C} / \mathrm{min}$ en atmósfera de Nitrógeno. Se obtuvieron los termogramas correspondientes, calculándose la temperatura de inicio de la degradación $\left(\mathrm{T}_{\mathrm{dTG}}\right)$ y la composición másica porcentual de los productos de la degradación. Posteriormente se interpretaron y compararon los termogramas resultantes.

Análisis de calorimetría de barrido diferencial (DSC): El objetivo de la implementación de este tipo de análisis fue medir la variación del flujo de calor asociado a las transiciones físicas y químicas en la secreción adhesiva de los onicóforos en función de la temperatura. Para el análisis se utilizó un equipo DSC Q10 V8.1 Build 261. Se analizaron muestras en estado líquido (3 $\mathrm{mg})$ y sólido $(0.1$ $\mathrm{mg}$ ) las cuales se sometieron a una velocidad de calentamiento de $10{ }^{\circ} \mathrm{C} / \mathrm{min}$ en atmósfera de Nitrógeno. Se calculó la temperatura correspondiente al flujo máximo de calor $\left(\mathrm{T}_{\mathrm{dmáx}}\right)$, temperatura y flujo de calor inicial y final de cada evento térmico y el área de flujo de calor $(\Delta \mathrm{H})$ de cada una de las transiciones.

Análisis de espectroscopía infrarroja (FTIR): Esta técnica se implementó para el análisis y caracterización de los componentes de la estructura secundaria de la secreción adhesiva. En un equipo FTIR-8400S, se analizó una muestra de secreción adhesiva en estado líquido. La muestra se preparó implementando el método de matriz de $\mathrm{KBr}$, que consiste en macerar la muestra con $\mathrm{KBr}$ hasta la homogenización. En la segunda derivada del espectro infrarrojo de la secreción adhesiva se amplió la región de la banda amida I comprendida entre 1700 y $1600 \mathrm{~cm}^{-1}$ con el fin de identificar los tipos característicos de la estructura secundaria de la proteína hélice alfa $(\alpha)$, láminas beta $(\beta)$ y giros $(\beta)$. Estos tipos estructurales se identificaron en el espectro infrarrojo teniendo como base las frecuencias de las longitudes de onda reportadas para cada uno de estos en la bibliografía. Se calculó el porcentaje de láminas $\beta$ presentes en la muestra implementando la formula de Gandhi (2006).

\section{RESULTADOS}

Especímenes: Quince onicóforos fueron recolectados dentro de los cafetales de la hacienda El Roble. Las medidas morfométricas realizadas a cada espécimen se registran en el Cuadro 2. A continuación se describe la especie a la que pertenecen los especímenes recolectados.

\section{CUADRO 2}

Valores morfométricos de Macroperipatus geagy vivos encontrados en los cafetales de la hacienda El Roble, Los Santos, Santander (Colombia)

TABLE 2

Morphometric values for live Macroperipatus geagy found at hacienda's coffee plantations El Roble, Los Santos, Santander (Colombia)

$\begin{array}{cccc}\text { Individuo } & \begin{array}{c}\text { Peso } \\ (\mathrm{g})\end{array} & \begin{array}{c}\text { Longitud } \\ (\mathrm{mm})\end{array} & \begin{array}{c}\text { Pares de } \\ \text { Lobópodos }\end{array} \\ 001 & 1.187 & 85 & 29 \\ 002 & 8.764 & 80 & 28 \\ 003 & 1.177 & 90 & 29 \\ 004 & 0.077 & 30 & 27 \\ 005 & 0.198 & 30 & 27 \\ 006 & 0.214 & 50 & 26 \\ 007 & 1.022 & 85 & 28 \\ 008 & 0.045 & 30 & 29 \\ 009 & 0.365 & 50 & 30 \\ 010 & 0.015 & 25 & 27 \\ 011 & 0.045 & 15 & 26 \\ 012 & 1.138 & 25 & 30 \\ 013 & 0.059 & 30 & 29 \\ 014 & 0.0131 & 30 & 28 \\ 015 & 0.021 & 30 & 29 \\ \text { Media }(\chi) & 0.956 & 45.666 & 28.133 \\ \text { Desv. Estándar } & 2.211 & 26.108 & 1.302 \\ (\mathrm{~S}) & 4.890 & 681.667 & 1.695 \\ \text { Varianza }\left(\mathrm{S}^{2}\right) & & & \end{array}$




\section{Macroperipatus geagy Bouvier 1899b}

Bouvier en 1907 describió un único tipo de M. geagy de la Guyana francesa. Posteriormente Clark en 1913 reportó para Colombia a $M$. geagy descrita a partir de dos hembras de $60 \mathrm{~mm}$ de longitud con 31 pares de lobópodos, cabeza y antenas extremadamente oscuras, pliegues dorsales transversales no separados por surcos definidos y con una banda ancha transversal más clara detrás de la región cefálica, colectadas en plantaciones de café en la Sierra Nevada de Santa Marta. En 1925 y 1941 Brues reportó una coloración parda rojiza en el cuerpo de especímenes de $M$. geagy de Panamá, sin reportar diferencias morfológicas específicas entre estas y las de Colombia reportadas por Clark en 1913.

Morfología: $M$. geagy presenta un número de lobópodos que varía entre 26 y 31 pares, siendo simétrico en la distribución de los lobópodos a cada lado del cuerpo. La longitud del

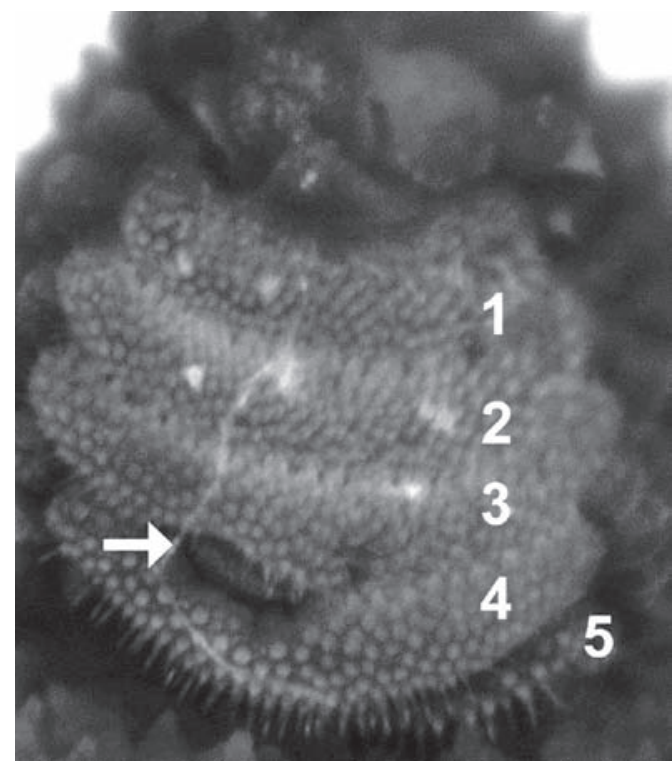

Fig. 1. Posición del tubérculo nefridial (flecha) y número de almohadillas de arrastre del lobópodo de $M$. geagy (100x).

Fig. 1. Position of the urinary tubercle (arrow) and number of creeping pads of $M$. geagy (100x). cuerpo varía entre 15 y $90 \mathrm{~mm}$ y el peso entre 0.013 y $8.764 \mathrm{~g}$ (Tabla 2). En el cuarto y quinto par de lobópodos la papila urinaria o tubérculo nefridial se ubica entre la línea divisoria de la tercera y cuarta almohadilla de arrastre del lobópodo sin dividir la cuarta almohadilla (Fig. 1), en algunos de los lobópodos se observa una pequeña proyección de la quinta almohadilla; cada lobópodo tiene tres papilas (dos anteriores y una posterior). La abertura genital se encuentra ubicada entre el penúltimo par de lobópodos (Fig. 2), característica de la familia Peripatidae. En cada segmento del cuerpo se observa un número variable (entre 10 y 12) de pliegues dorsales transversales al eje del cuerpo, divididos por surcos profundos y espaciosos, entre 7 y 9 de estos pliegues terminan o se bifurcan en la zona de inicio de los lobópodos. La base de las papilas dorsales del cuerpo es cuadrada, característica distintiva del género; las papilas dorsales primarias tienen forma de seno globoso que termina en una estructura cónica alargada con una cerda sensorial en el extremo (Fig.

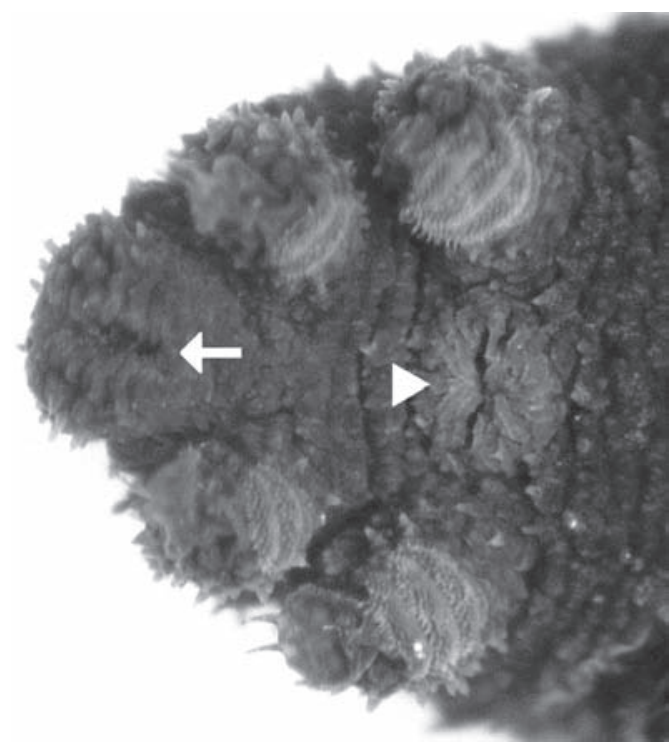

Fig. 2. Ubicación de la abertura genital (cabeza de la flecha) y ano (flecha) de M. geagy (200x).

Fig. 2. Position of the genital opening (arrow's head) and anus (arrow) of M. geagy (200x). 

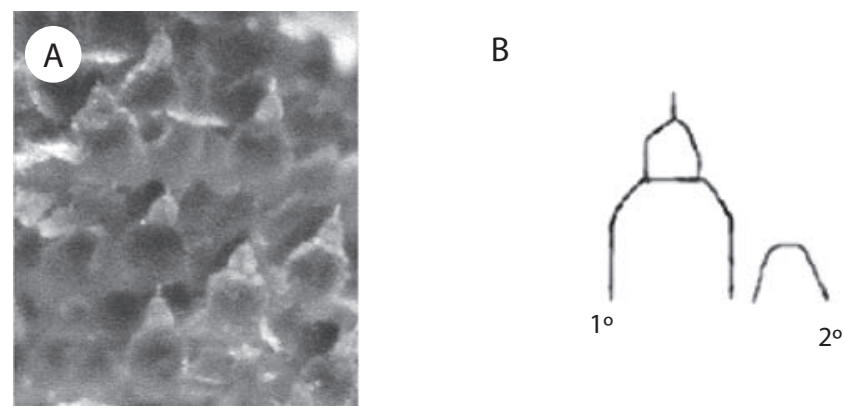

Fig. 3. (A.) Superficie dorsal del cuerpo; (B.) Detalle de las papilas primarias y accesorias o secundarias del dorso del cuerpo de $M$. geagy (400x).

Fig. 3. (A.) Dorsal surface of the body; (B.) Detail of the primary and accesory papillae on the back of the body of $M$. geagy (400x).

3); las papilas dorsales accesorias o secundarias al igual que las papilas primarias tienen forma de seno pero no todas terminan en la estructura cónica alargada con cerda sensorial, son de menor tamaño y se distribuyen entre las papilas primarias sin formar un patrón determinado. En la región anterior a ambos lados de la boca se ubican las glándulas de la seda, las antenas y los ojos (Fig. 4); las antenas de coloración más oscura en comparación con el resto del cuerpo y con un número de segmentos o anillos que varia entre 35 y 40 ; la boca rodeada de papilas carnosas alberga el labrum y las mandíbulas.
Como carácter diagnostico $M$. geagy presenta un diente principal, un diente accesorio y siete dentículos en la mandíbula interna (Fig. 5A, B). La línea dorsal media del cuerpo esta formada por papilas de igual tamaño (Fig. 6) y de coloración más oscura que las demás papilas primarias y accesorias del dorso, generalmente sin terminación cónica y sin cerda sensorial.

Reconstrucción 3D de la superficie dorsal de M. geagy: En la reconstrucción de la superficie dorsal del cuerpo de $M$. geagy (Fig. 6) las papilas primarias y accesorias se
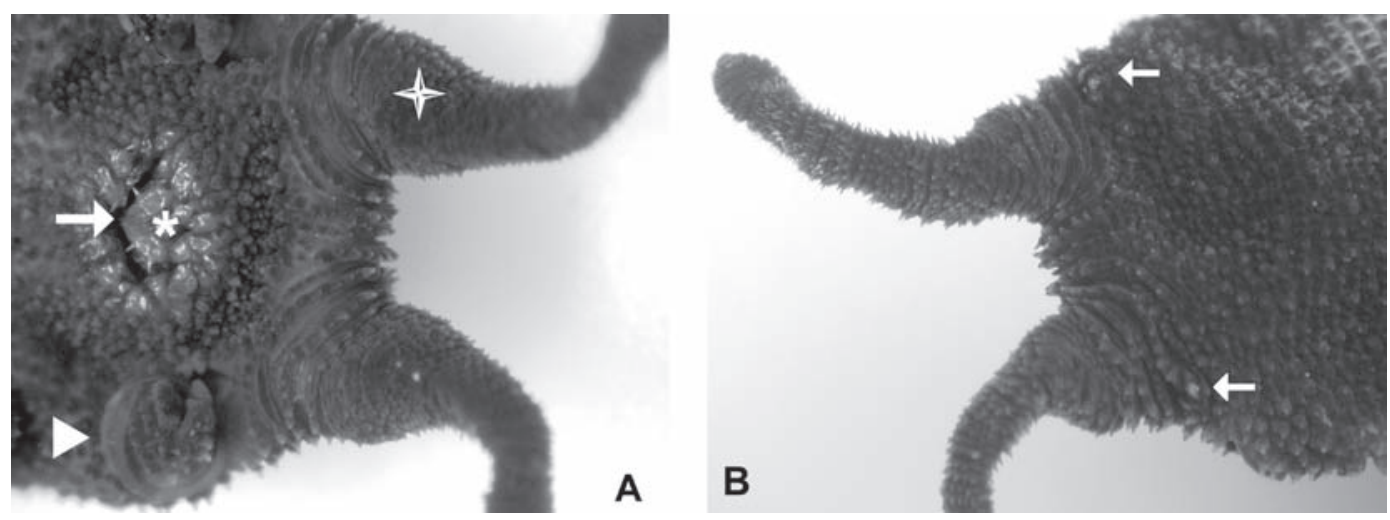

Fig. 4. (A.) Región anterior ventral, antena (cruz), glándula de la seda (cabeza de la fecha) y boca (flecha), dentro de la boca se observa el labrum (asterisco) de M. geagy (100x); (B.) Región anterior dorsal de M. geagy, ojos (flechas) (100x).

Fig. 4. (A.) Ventral side of the body, antennae (croos), slime gland (arrow's head) and mouth (arrow), inside the mouth is observed the labrum (asterisk) of M. geagy (100x); (B.) Dorsal side of the body of M. geagy, eyes (arrows) (100x). 

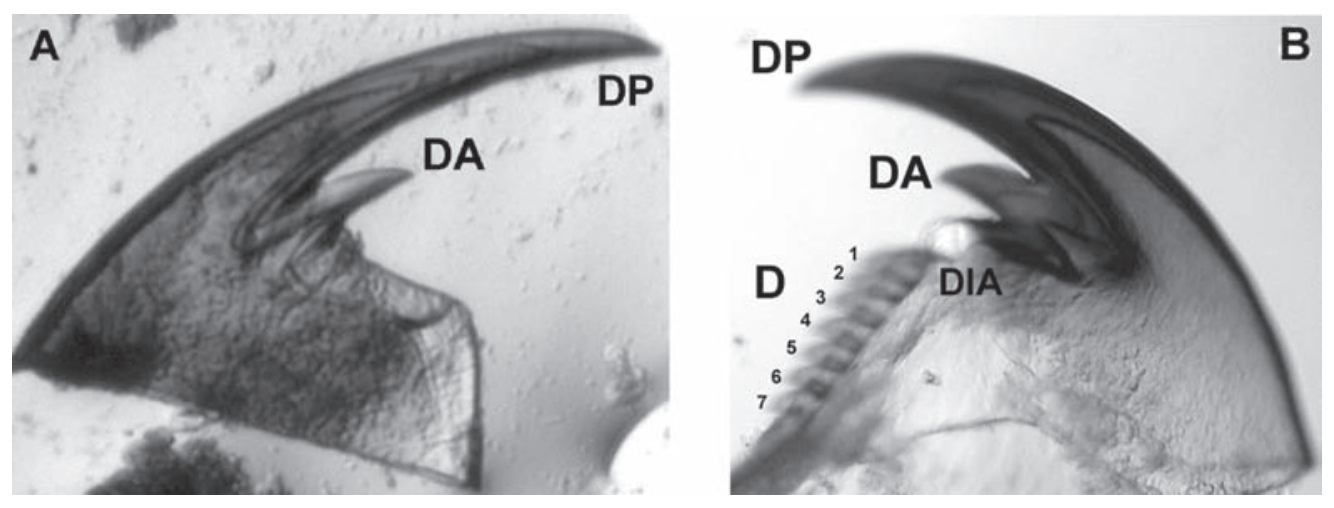

Fig. 5. (A.) Mandíbula externa; (B.) Mandíbula interna de M. geagy. DP: diente principal, DA: diente accesorio, DIA: diastema, D: dentículos (10x).

Fig. 5. (A.) Outer jaw; (B.) Inner jaw of M.geagy. DP: main tooth, DA: accesory teeth, DIA: diastema, D: denticles (10x).

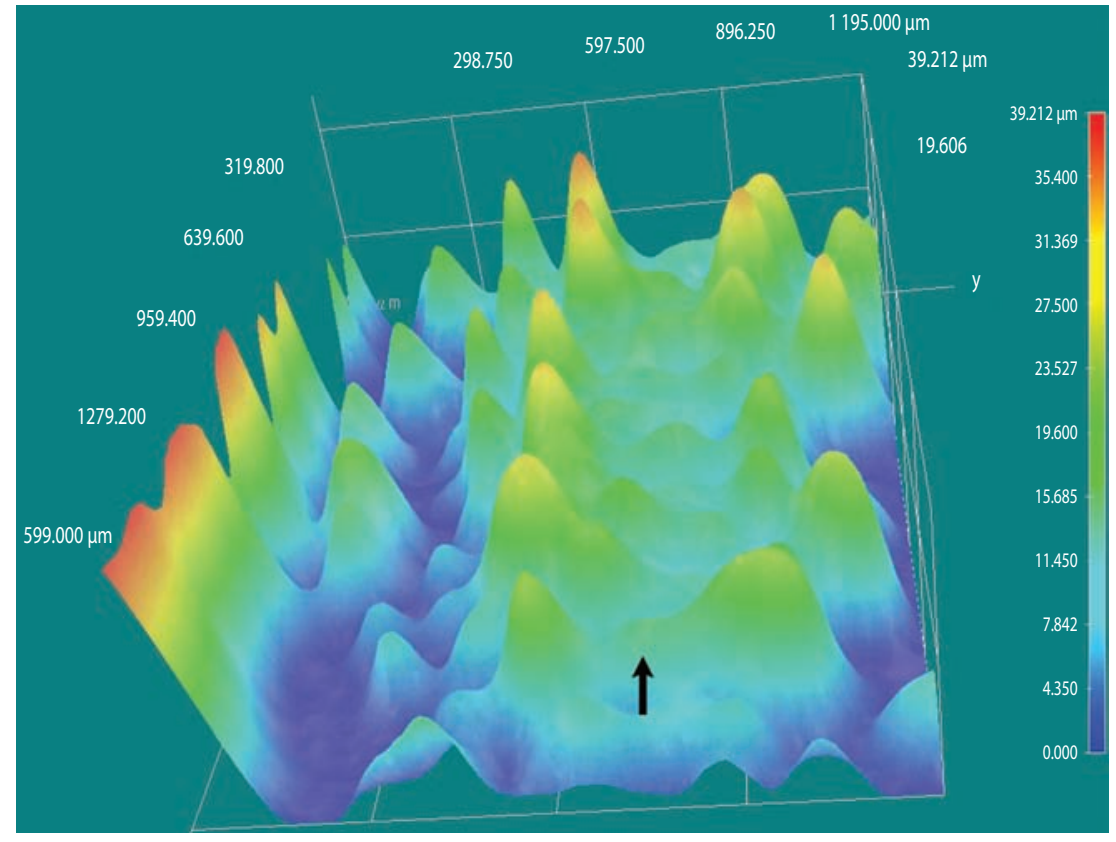

Fig. 6. Reconstrucción 3D de la superficie dorsal de M. geagy, línea dorsal media (flecha).

Fig. 6. Reconstruction 3D of the dorsal surface of $M$. geagy, line dorsal half (arrow). 
observan distribuidas de manera no uniforme, sin establecer un patrón de distribución definido. La línea dorsal media del onicóforo la conforman papilas de tamaño pequeño (19 $\mu \mathrm{m})$ que en algunas zonas de la línea tratan de distribuirse en pares. A ambos lados de la línea media se observan papilas de mayor tamaño (entre 35 y $25 \mu \mathrm{m}$ ) de las cuales, la mayoría alcanzan la altura máxima observada en las papilas del dorso de $M$. geagy: 35 y $39 \mu \mathrm{m}$. Las demás papilas de la superficie del cuerpo, las primarias y las accesorias presentan alturas oscilantes entre los 23 y $31 \mu \mathrm{m}$ y los 11 y 19 $\mu \mathrm{m}$, respectivamente.

Historia natural de $M$. geagy en los cafetales de la Mesa de Los Santos: Los onicóforos de esta especie los encontramos restringidos a microhábitats húmedos y oscuros, dentro de galerías de los troncos en descomposición, debajo de la hojarasca del cafetal y dentro de montículos de tierra húmedos, con poca exposición a la luz solar. Habitando en grupos de 8 a 12 individuos entre jóvenes y adultos. Los jóvenes frecuentemente caminaban sobre el dorso de los onicóforos más grandes y cuando el grupo fue expuesto a la luz solar, en el momento en que el tronco donde habitan fue abierto, se aglomeraron unos sobre otros; según Ruhberg (1985) este gregarismo es típico del grupo. El patrón característico de pigmentación del cuerpo observado en especímenes adultos vivos fue café ceniza (Fig. 7), oscuro en el dorso, con la cabeza y antenas más oscuras, y con el vientre más claro y con menor número de papilas; en los jóvenes la pigmentación se observó más clara en dorso y vientre, aunque algunos mostraron una coloración café rojiza, la apariencia observada en los jóvenes fue menos aterciopelada (menor desarrollo y pigmentación de las papilas) en comparación con los adultos; en especímenes conservados en alcohol del 70\% la coloración se tornó café oscura en el dorso, las antenas y la parte externa de los lobópodos, en el vientre y en la superficie interna de los lobópodos la coloración era más clara.

La respuesta inmediata de los onicóforos ante cualquier estímulo externo fue expulsar la secreción adhesiva desde las papilas orales, esta expulsión de los onicóforos adultos alcanzó distancias de $30 \mathrm{~cm}$ aproximadamente. Los onicóforos de mayor tamaño expulsaron mayor cantidad de secreción en una sola oportunidad. En el momento de la expulsión la secreción se observó casi transparente y de consistencia líquida formando hilos en los que se observaron pequeñas gotas de secreción levemente espaciadas a lo largo de estos. Después de estar en contacto con el aire, la secreción tomó una coloración blancuzca y se solidificó siendo menos flexible y adhesiva. En algunos de los onicóforos en especial los de mayor tamaño, se observaron cicatrices en el dorso del cuerpo y mutilación de algunos de los lobópodos, esto podría ser el resultado de enfrentamientos con los posibles depredadores como alacranes y serpientes también observados en el hábitat. Dentro del microhábitat de $M$. geagy también observamos otros pequeños invertebrados como isópodos, larvas de coleópteros, lombrices, babosas y termitas, que constituirían la base de la alimentación de estos onicóforos. Algunos de los onicóforos recolectados los conservamos vivos en terrarios observando que el proceso de muda de la cutícula (ecdisis) ocurría a intervalos irregulares de tiempo.

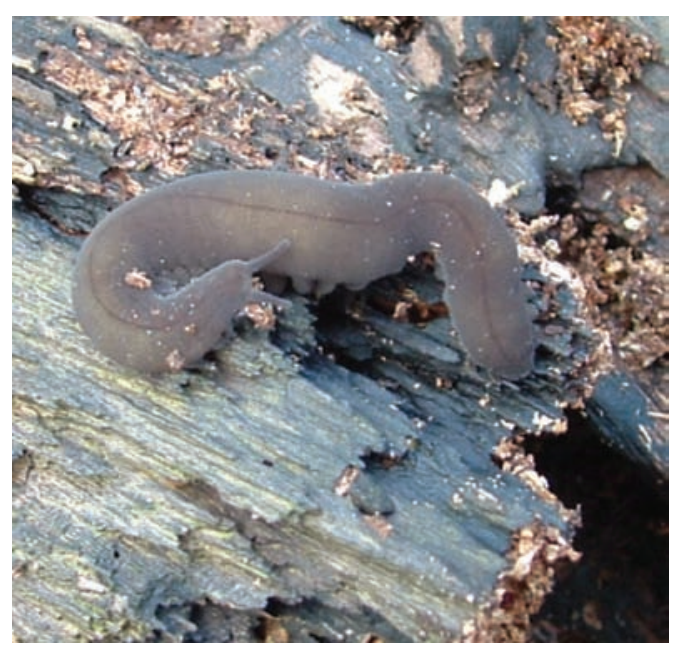

Fig. 7. Apariencia externa de M. geagy en el hábitat natural.

Fig. 7. Aspect of M. geagy in natural habitat. 
A continuación se presenta una clave para algunas de las especies de Macroperipatus (Peripatidae) distribuidas en el Caribe y sureste de América del sur. Existen otras especies del Caribe como M. guianensis (Guyana), M. trinidadensis (Trinidad), M. insularis (Haití) y $M$. clarki (Jamaica) de las que se tiene poco conocimiento, de estas solo se mencionan algunos caracteres morfológicos en manuscritos comparativos publicados de las especies de Costa Rica. De acuerdo con lo anterior estas especies no se incluyen en la clave.

\section{Clave para algunas especies de Macroperipatus Clark 1913 (Peripatidae)}

Diagnosis: Macroperipatus se caracteriza morfológicamente por poseer entre 26 y 34 pares de lobópodos; tres papilas en cada lobópodo (dos anteriores y una posterior); ubicación de los tubérculos nefridiales en el cuarto y quinto par de lobópodos, abriendo espacio entre la tercera y cuarta almohadilla de arrastre del lobópodo; con doce pliegues transversales en el dorso de cada segmento del cuerpo; abertura genital o gonóporo ubicado entre el penúltimo par de lobópodos; papilas primarias de la superficie dorsal con base cuadrangular y como característica única de la familia Peripatidae, pigmentos del cuerpo solubles en etanol.

1a. Papilas accesorias de la superficie dorsal del cuerpo ausentes $\ldots \ldots \ldots \ldots \ldots$ M. perrieri

1b. Papilas accesorias de la superficie dorsal del cuerpo presentes. $\ldots \ldots \ldots \ldots \ldots \ldots \ldots .2$

2a. Con proyección de la quinta almohadilla de arrastre en el lobópodo $\ldots \ldots \ldots \ldots \ldots \ldots$

2b. Sin proyección de la quinta almohadilla de arrastre, solo cuatro almohadillas de arrastre en el

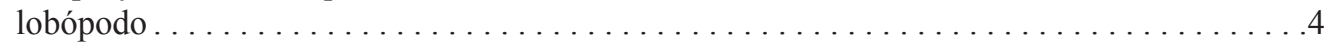

3a. Un diente accesorio en la mandíbula interna y siete dentículos en la sierra de la mandíbula

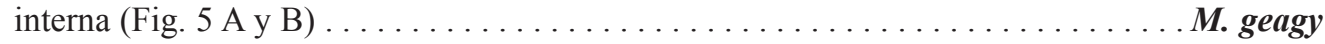

3b. Dos dientes accesorios en la mandíbula interna (Fig. 8 A y B) $\ldots \ldots \ldots \ldots \ldots \ldots \ldots . \ldots$

4a. 29 a 32 pares de lobópodos y 36 anillos en la antena M. machadoi

4b. 24 a 29 pares de lobópodos y 38 anillos en la antena M. acacioi

5a. Primer diente accesorio de la mandíbula interna más desarrollado y doce dentículos en la sierra

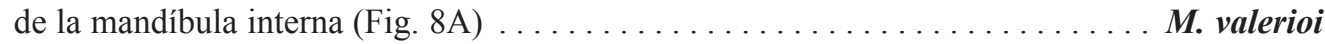

5b. Segundo diente accesorio de la mandíbula interna desarrollado (Fig. 8B) . . . . M. torquatus

Análisis termogravimétrico (TG): En la historia térmica de la muestra líquida de la secreción adhesiva de M. geagy (Fig. 9) se observan cuatro etapas según los cambios en el porcentaje del peso de la muestra en función de la temperatura. La primera etapa se caracterizó por la pérdida de agua fisisorbida en la que se perdió cerca del $60 \%$ del peso total de la muestra al alcanzar los $70^{\circ} \mathrm{C}$, esta etapa en el análisis calorimétrico se observó en un pico 


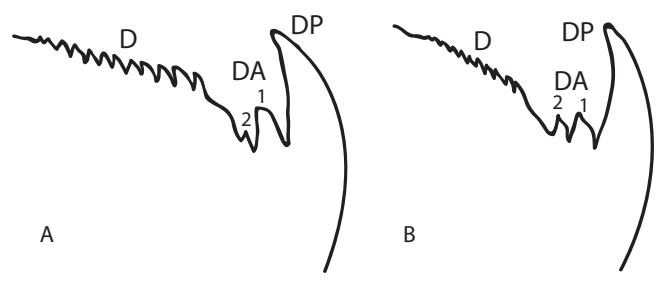

Fig. 8. (A.) Mandíbula interna de M. valerioi; (B.) Mandíbula interna de $M$. torquatos, redibujado de Morera y León 1986.

Fig. 8. (A.) Inner jaw of M. valerioi; (B.) Inner jaw of $M$. torquatos, redraws of Morera and León 1986.

endotérmico entre los 26 y $93{ }^{\circ} \mathrm{C}$. Entre los 100 y $280{ }^{\circ} \mathrm{C}$ la pérdida del peso presenta una leve estabilización (II etapa), contrastando con la tercera fase del comportamiento térmico de la muestra en la que se observó la pérdida del $88 \%$ del peso total de la muestra entre los 280 y $355^{\circ} \mathrm{C}$. La temperatura del inicio de la degradación $\left(\mathrm{T}_{\mathrm{dTG}}\right)$ se registró a los $355{ }^{\circ} \mathrm{C}$ extendiéndose hasta los $485{ }^{\circ} \mathrm{C}$ dejando un residuo del $7.5 \%(0.74 \mathrm{mg})$ del peso total de la muestra $(9.894 \mathrm{mg})$.

En el análisis de la muestra de secreción adhesiva en estado sólido (Fig. 10) se registró una pérdida inicial del 13\% del peso total de la muestra en la etapa inicial del calentamiento cerca de los $90{ }^{\circ} \mathrm{C}$, atribuida a la pérdida de agua en la muestra. En el rango de los 100 y $205{ }^{\circ} \mathrm{C}$ se observó una leve estabilización en la pérdida de peso en la muestra (etapa II). En la tercera etapa del comportamiento térmico de la secreción comprendida entre los 220 y $350{ }^{\circ} \mathrm{C}$ se observó un rápido descenso del porcentaje del peso de la muestra, perdiéndose el $65 \%$ del peso total. Finalmente la muestra inició su etapa de descomposición $\left(\mathrm{T}_{\mathrm{dTG}}\right)$ a los $365{ }^{\circ} \mathrm{C}$ dejando un residuo del $17 \%(0.03 \mathrm{mg})$ del peso inicial de la muestra a los $495{ }^{\circ} \mathrm{C}$.

Análisis calorimétrico (DSC): La muestra de secreción adhesiva en estado líquido se caracterizó por presentar un evento endotérmico el

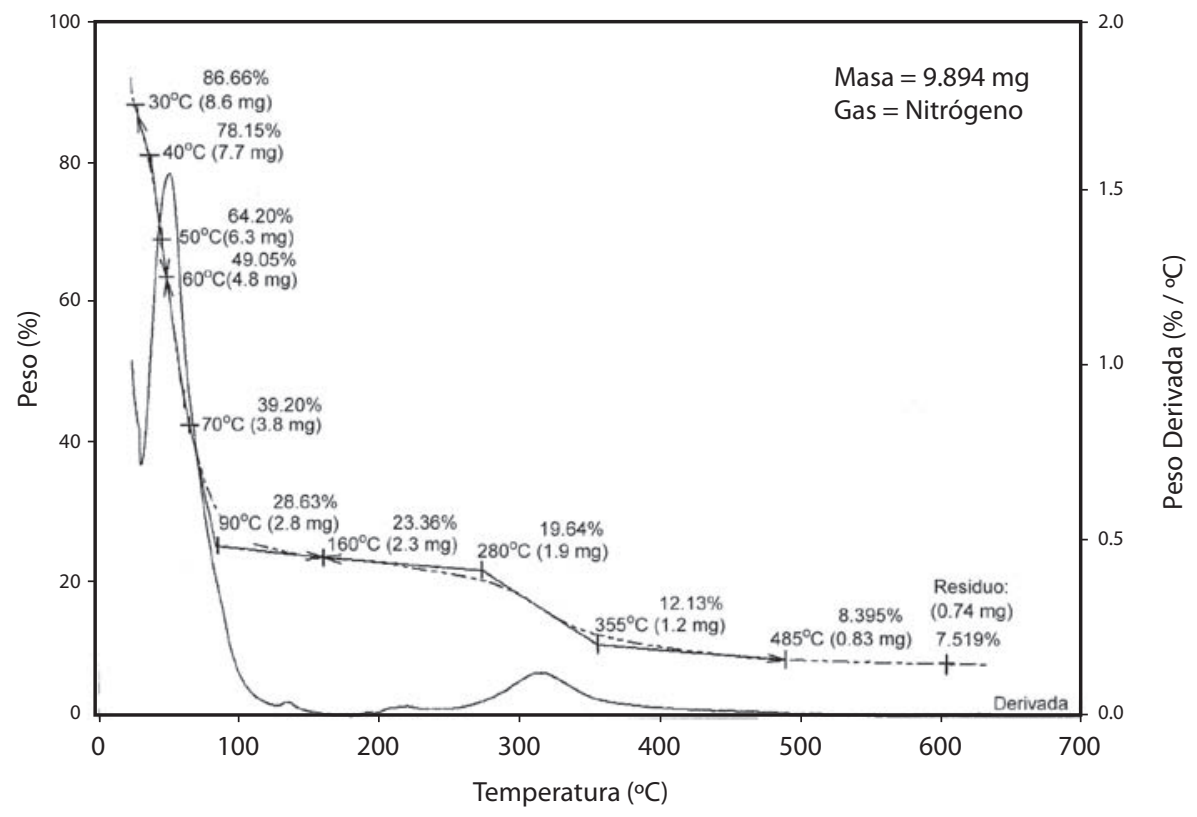

Fig. 9. Termograma de la secreción adhesiva de M. geagy en estado líquido en atmósfera de nitrógeno.

Fig. 9. Thermogram of the adhesive secretion of M. geagy in liquid state in nitrogen atmosphere. 


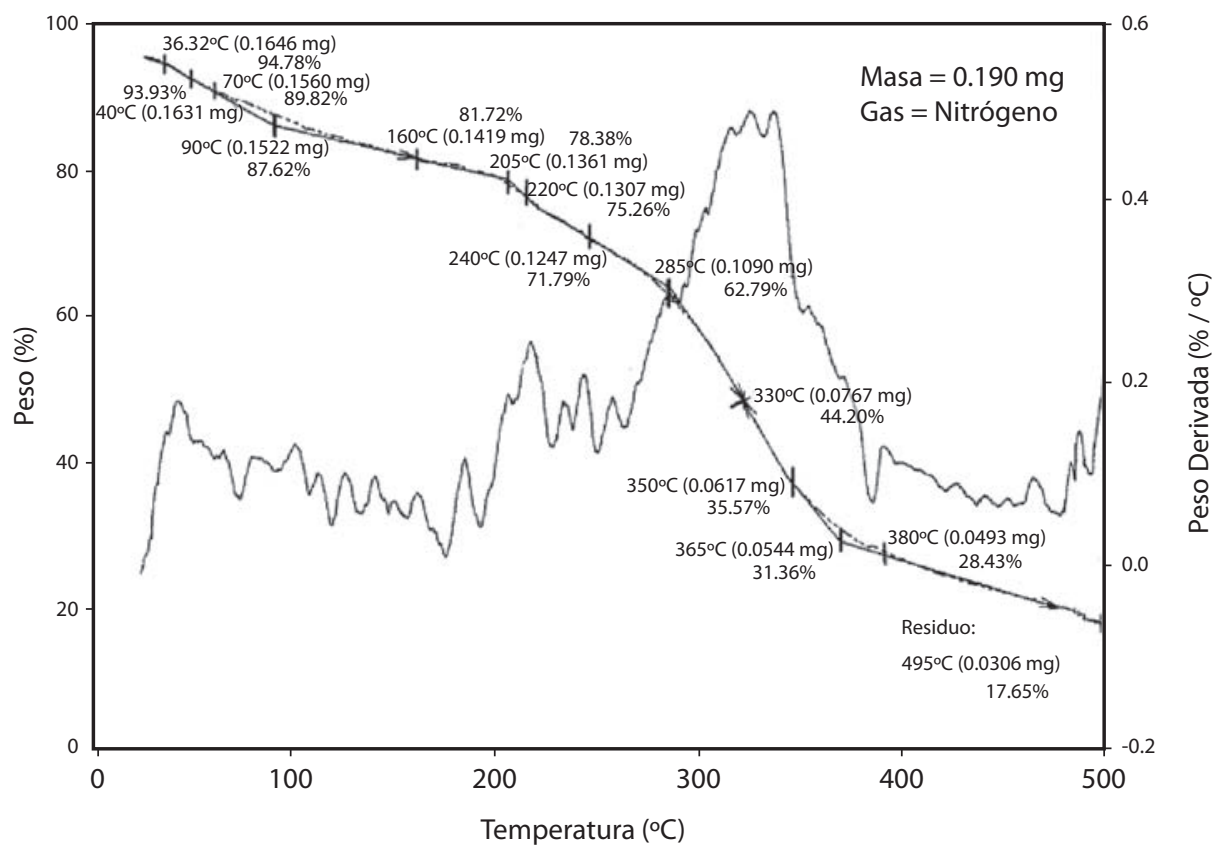

Fig. 10. Termograma de la secreción adhesiva de M. geagy en estado sólido en atmósfera de nitrógeno.

Fig. 10. Thermogram of the adhesive secretion of M. geagy in solid state in nitrogen atmosphere.

cual inició con un flujo de calor de $-0.28 \mathrm{~W} / \mathrm{g}$ a los $26{ }^{\circ} \mathrm{C}$ finalizando cerca de $\operatorname{los} 93{ }^{\circ} \mathrm{C}$ con un flujo de calor de $-0.082 \mathrm{~W} / \mathrm{g}$ (Fig. 11). El máximo flujo de calor alcanzado en el evento fue de $-1.83 \mathrm{~W} / \mathrm{g}$ a una temperatura máxima $\left(\mathrm{T}_{\text {dmáx }}\right)$ de $62{ }^{\circ} \mathrm{C}$. El área de flujo de calor $(\Delta \mathrm{H})$ del evento fue de $-1078 \mathrm{~J} / \mathrm{g}$ (ver Cuadro 3).

El termograma que representa el flujo de calor en la muestra sólida de la secreción de M. geagy se caracterizó por presentar un pico endotérmico inicial y un pico exotérmico y endotérmico en su etapa final (Fig. 12). El primer evento de absorción de calor inició cerca de los $27{ }^{\circ} \mathrm{C}$ con un flujo de calor de $-3.78 \mathrm{~W} / \mathrm{g}$ finalizando en los $119{ }^{\circ} \mathrm{C}$ con un flujo de calor de $-7.64 \mathrm{~W} / \mathrm{g}$. El flujo máximo de calor registrado fue de $-8.83 \mathrm{~W} / \mathrm{g}$ a una temperatura máxima $\left(\mathrm{T}_{\mathrm{dmáx}}\right)$ de $76{ }^{\circ} \mathrm{C}$, el área de flujo de calor $(\Delta \mathrm{H})$ calculado para este evento fue de $-794 \mathrm{~J} / \mathrm{g}$. Después de los $243{ }^{\circ} \mathrm{C}$ la muestra presentó una fase de liberación de energía con un flujo de calor máximo de calor de -10.90
W/g a una temperatura máxima $\left(\mathrm{T}_{\text {dmáx }}\right)$ de 257 ${ }^{\circ} \mathrm{C}$ abarcando un área de flujo de calor $(\Delta \mathrm{H})$ de $132.2 \mathrm{~J} / \mathrm{g}$. El tercer y último evento registrado en el termograma fue endotérmico en el que la temperatura máxima del flujo de calor $\left(\mathrm{T}_{\text {dmáx }}\right)$ calculada fue de $316^{\circ} \mathrm{C}$ siendo el flujo de calor máximo de $-17.6 \mathrm{~W} / \mathrm{g}$. El área del flujo de calor $(\Delta \mathrm{H})$ calculada para este evento fue de $-744 \mathrm{~J} / \mathrm{g}$ (ver Cuadro 3). El flujo de calor en la muestra tiende a estabilizarse manteniéndose en -7.85 $\mathrm{W} / \mathrm{g}$ entre los 315 y $383{ }^{\circ} \mathrm{C}$.

Análisis de infrarrojos (FTIR): Sobre la segunda derivada del espectro infrarrojo de la secreción adhesiva de $M$. geagy se observa la ampliación de la banda amida I comprendida entre el rango de frecuencias de 1700 y 1600 $\mathrm{cm}^{-1}$ (Fig. 13). Dentro de esta región se encuentran los tipos de conformaciones característi$\cos$ de la estructura secundaria de la proteína, conformación hélice alfa $(\alpha)$, lamina beta $\mathrm{y}$ giros beta $(\beta)$ (Fig. 14 y Cuadro 4) según las 


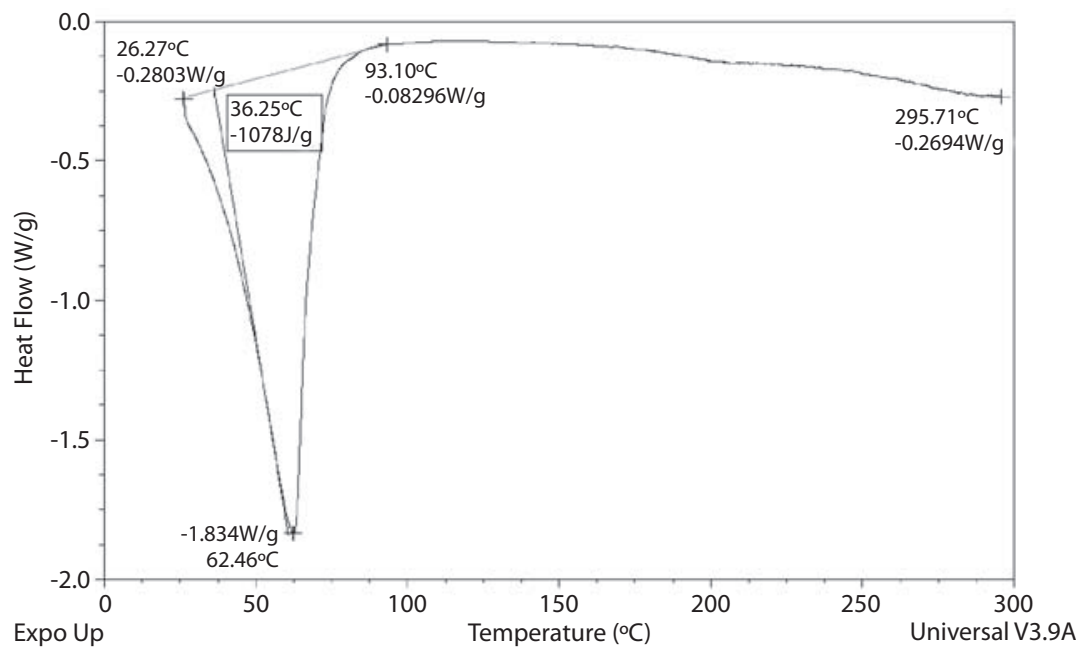

Fig. 11. Termograma (DSC) de la secreción adhesiva de $M$. geagy en estado líquido. Los valores dentro del recuadro corresponden al área de flujo de calor $(\Delta \mathrm{H})$ del evento endotérmico.

Fig. 11. Thermogram (DSC) of the adhesive secretion of $M$. geagy in state liquid. The values inside the box correspond to the area of heat flow $(\Delta \mathrm{H})$ of the event endothermic.

\section{CUADRO 3}

Flujo de calor en la secreción adhesiva de M. geagy en estado líquido y sólido

TABLE 3

Flow of heat in the adhesive secretion of M. geagy in liquid and solid

\begin{tabular}{|c|c|c|c|c|c|}
\hline \multicolumn{2}{|l|}{ Muestra } & \multicolumn{2}{|l|}{ Sólida } & \multicolumn{2}{|c|}{ Líquida } \\
\hline \multicolumn{2}{|l|}{ Peso } & \multicolumn{2}{|l|}{$0.1 \mathrm{mg}$} & \multicolumn{2}{|l|}{$3 \mathrm{mg}$} \\
\hline Evento térmico & Características del evento & & & & \\
\hline \multirow[t]{5}{*}{ Endotérmico } & Flujo máximo de calor & \multicolumn{2}{|c|}{$-8.839 \mathrm{~W} / \mathrm{g}$} & \multicolumn{2}{|c|}{$-1.834 \mathrm{~W} / \mathrm{g}$} \\
\hline & $\left(\mathrm{T}_{\text {dmáx }}\right)$ & \multicolumn{2}{|l|}{$76^{\circ} \mathrm{C}$} & \multicolumn{2}{|l|}{$62{ }^{\circ} \mathrm{C}$} \\
\hline & $(\Delta \mathrm{H})$ & \multicolumn{2}{|c|}{$-794.6 \mathrm{~J} / \mathrm{g}$} & \multicolumn{2}{|c|}{$-1078 \mathrm{~J} / \mathrm{g}$} \\
\hline & Temperatura inicial y Flujo de calor inicial & $27^{\circ} \mathrm{C}$ & $-3.783 \mathrm{~W} / \mathrm{g}$ & $26{ }^{\circ} \mathrm{C}$ & $-0.2803 \mathrm{~W} / \mathrm{g}$ \\
\hline & Temperatura inicial y Flujo de calor inicial & $119^{\circ} \mathrm{C}$ & $-7.649 \mathrm{~W} / \mathrm{g}$ & $93{ }^{\circ} \mathrm{C}$ & $-0.0829 \mathrm{~W} / \mathrm{g}$ \\
\hline \multirow[t]{5}{*}{ Exotérmico } & Flujo máximo de calor & \multicolumn{4}{|c|}{$-10.90 \mathrm{~W} / \mathrm{g}$} \\
\hline & $\left(\mathrm{T}_{\mathrm{dmáx}}\right)$ & \multicolumn{4}{|c|}{$257^{\circ} \mathrm{C}$} \\
\hline & $(\Delta \mathrm{H})$ & \multicolumn{4}{|c|}{$132.2 \mathrm{~J} / \mathrm{g}$} \\
\hline & Temperatura y flujo de calor inicial & $242{ }^{\circ} \mathrm{C}$ & $-11.37 \mathrm{~W} / \mathrm{g}$ & & \\
\hline & Temperatura y flujo de calor final & $269^{\circ} \mathrm{C}$ & $-13.18 \mathrm{~W} / \mathrm{g}$ & & \\
\hline \multirow[t]{5}{*}{ Endotérmico } & Flujo máximo de calor & \multicolumn{4}{|c|}{$17.68 \mathrm{~W} / \mathrm{g}$} \\
\hline & $\left(\mathrm{T}_{\mathrm{dmáx}}\right)$ & \multicolumn{4}{|c|}{$316^{\circ} \mathrm{C}$} \\
\hline & $(\Delta \mathrm{H})$ & \multicolumn{4}{|c|}{$-744.9 \mathrm{~J} / \mathrm{g}$} \\
\hline & Temperatura y flujo de calor inicial & $286^{\circ} \mathrm{C}$ & $-14.66 \mathrm{~W} / \mathrm{g}$ & & \\
\hline & Temperatura y flujo de calor final & $383{ }^{\circ} \mathrm{C}$ & $-17.83 \mathrm{~W} / \mathrm{g}$ & & \\
\hline
\end{tabular}

$\left(\mathrm{T}_{\mathrm{dmáx}}\right)$ : Temperatura máxima de flujo de calor.

$(\Delta \mathrm{H})$ : Área de flujo de calor. 


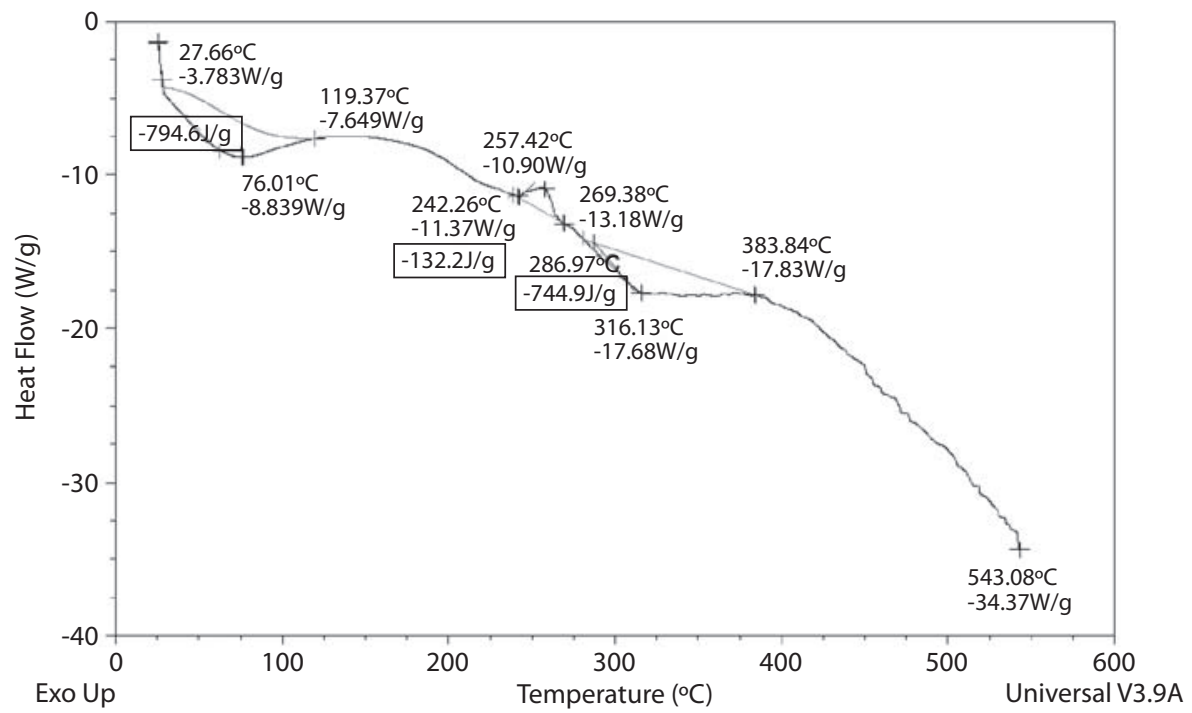

Fig. 12. Termograma (DSC) de la secreción adhesiva de M. geagy en estado sólido. Los valores dentro del recuadro corresponden a las áreas de flujo de calor $(\Delta \mathrm{H})$ de los eventos térmicos.

Fig. 12. Thermogram (DSC) of the adhesive secretion of $M$. geagy in state solid. The values inside the box correspond to the area of heat flow $(\Delta \mathrm{H})$ of the event thermic.

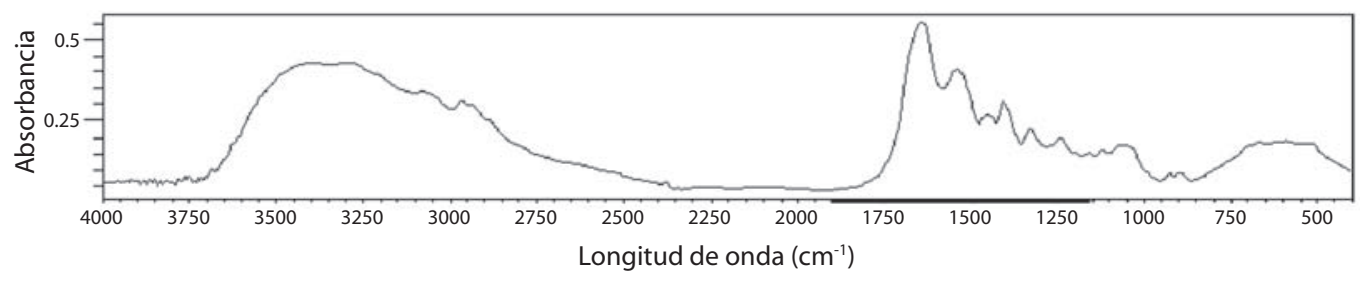

Fig. 13. Segunda deriva del espectro infrarrojo de la secreción adhesiva de M. geagy.

Fig. 13. Second derived from the infrared spectrum of adhesive secretion of M. geagy.

frecuencias de longitudes de onda reportadas en la bibliografía para cada uno de estos tipos de conformación en diferentes proteínas (ver Chirgadze et al. 1975, Venyaminov y Kalnin 1990, Dong et al. 1990). Utilizando los datos del área bajo la curva correspondiente a los picos de 1630 y $1650 \mathrm{~cm}^{-1}$ e implementando la formula matemática de Gandhi (2006) se calculó el porcentaje de láminas $(\beta)$ presentes en la muestra de la secreción adhesiva de $M$. geagy, así:
$\%$ de lamina $\beta$ en la región amida $I=[($ Área bajo la curva del pico de 1630) / (Área bajo la curva del pico de $1650+$ Área bajo la curva del pico de 1630)] X 100.

El porcentaje de laminas $\beta$ presente en la muestra de la secreción adhesiva de $M$. geagy fue del $59 \%$.

$\%$ de lamina $\beta$ en la región amida I de la secreción 


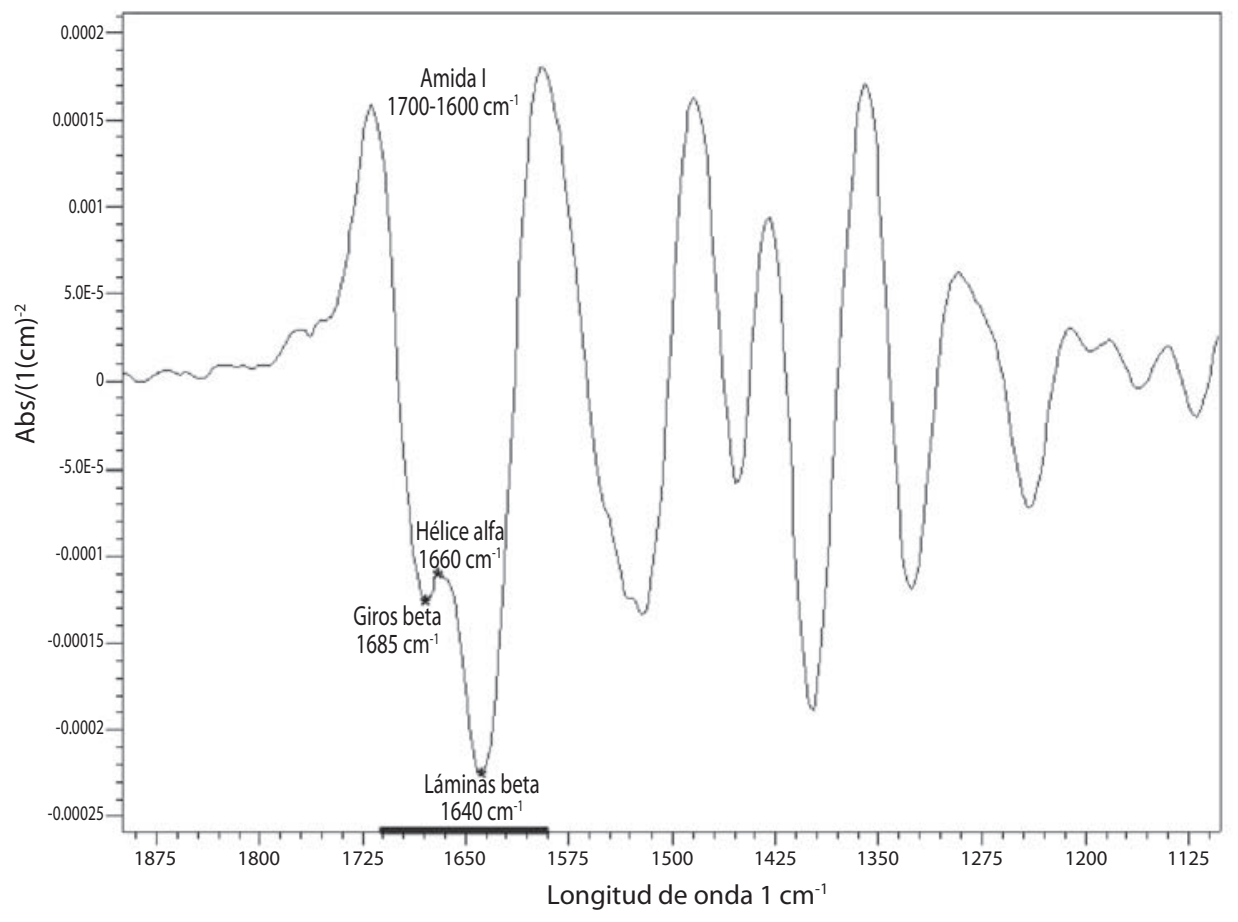

Fig. 14. Ampliación de la región de la banda amida I de la segunda derivada del espectro infrarrojo de la secreción adhesiva de M. geagy.

Fig. 14. Enlargement of the region of the amide I band of the second derivate of the infrarred specter of the adhesive secretion of M. geagy.

adhesivadeM.geagy $=[10.237 /(6.992+10.237)]$ x $100=\mathbf{5 9 . 4 1 7 2 \%}$

\section{DISCUSIÓN}

Los onicóforos de los géneros Macroperipatus, Epiperipatus y Peripatus reportados para Colombia se caracterizan morfológicamente por presentar tres papilas en cada lobópodo (dos anteriores y una posterior), tubérculos nefridiales del cuarto y quinto par de lobópodos ubicados debajo de la tercera almohadilla de arrastre del lobopodo y por poseer doce pliegues en cada segmento del cuerpo (Peck 1975). En 1907 Bouvier en su

\section{CUADRO 4}

Frecuencias de longitud de onda dentro la de la banda amida I de los diferentes tipos de conformación estructural en la secreción adhesiva de M. geagy

TABLE 4

Frequency wavelength within of the amide I band of the different kinds of structural conformation in adhesive secretion of $\mathrm{M}$. geagy

\begin{tabular}{|cc|}
\hline Frecuencia $\left(\mathrm{cm}^{-1}\right)^{*}$ & Estructura Secundaria \\
\hline $1600 \pm 2.0$ & Hélice $\alpha$ \\
$1640 \pm 1.0$ & Lamina $\beta$ \\
$1685 \pm 0.2$ & Giros $\beta$
\end{tabular}

${ }^{*} \mathrm{~cm}^{-1}$ : Unidad de longitud de onda. 
"Monographie des Onychophores" describió a $M$. geagy de la costa de Guyana, más tarde la especie fue reportada por Clark en Panamá en 1913 y en las montañas de Santa Marta Colombia en 1925 no encontrando diferencias morfológicas entre los especímenes de los dos países, observo en la superficie dorsal del cuerpo marcas de forma cuadrangular similares a cápsulas o tabletas poco notables cuando el cuerpo del animal estaba extendido. Esta última característica descrita por Clark es la que actualmente caracteriza al género. La base de las papilas del dorso del cuerpo de las especies de Macroperipatus presenta una forma cuadrangular (Peck 1975). Morera-Brenes y León en 1986 describieron a M. valerioi de Costa Rica, esta especie presenta características morfológicas similares a las observadas en M. geagy. Dos dientes accesorios en la mandíbula interna el primero con mayor desarrollo que el segundo y 13 dentículos en la sierra de la mandíbula interna (Fig. 8A) caracterizan la mandíbula de $M$. valerioi contrastando con la morfología de la mandíbula interna de $M$. geagy que presenta un diente accesorio y 7 dentículos en la sierra de la mandíbula interna (Fig. 5), el tubérculo nefridial que en $M$. valerioi se presenta libre y ubicado hacia un costado entre la tercera y cuarta almohadilla de arrastre del lobopodo, esta última almohadilla se presenta delgada y curvada en $M$. geagy donde el tubérculo nefridial se observa profundamente hundido entre la línea divisoria de la tercera y cuarta almohadilla, centrado y sin curvar o dividir las almohadillas, en esta especie también se observa una pequeña proyección de la quinta almohadilla (Fig. 1), carácter que comparte con $M$. torquatus y $M$. perrieri. Geográficamente M. geagy (Guyana, Panamá y Colombia) es cercana a $M$. torquatus (Trinidad) y $M$. perrieri (México).

En 1966 Peck recolectó en Costa Rica un onicóforo al que determino como M. geagy (Morera-Brenes y León 1986), posteriormente Morera-Brenes y León en 1986 después de observar y determinar a $M$. valerioi y teniendo en cuenta la baja dispersión y el reducido flujo génico de estos invertebrados en amplios rangos geográficos, sugirieron que el espécimen recolectado por Peck podría ser $M$. valerioi. Clark a principios del siglo XIX ya identificaba la facilidad de cometer errores cuando de la determinación o sistemática de los onicóforos se trataba atribuyéndolos a la ausencia de claves para las especies existentes. Existen algunas descripciones morfológicas originales de onicóforos del Caribe en las que se mencionan ciertos caracteres de las especies reportadas para América del Sur (MoreraBrenes y León 1986). Para evitar posibles errores en la sistemática de los onicóforos en futuras investigaciones se recomienda tener en cuenta caracteres como la morfología de la mandíbula interna y externa. En cuanto al hábitat, los onicóforos del Caribe y Sur América optan por el mismo tipo, hábitats húmedos, bajo la hojarasca de lugares sombríos y dentro de troncos en descomposición, a menudo en áreas poco intervenidas, lo que permite identificar no solo la ubicación y posible distribución de los onicóforos en el área sino alcanzar un conocimiento más profundo en cuanto a la biología de estos fósiles vivientes.

El comportamiento térmico de la secreción adhesiva en estado líquido de $M$. geagy se caracterizó en la primera etapa del calentamiento $\left(25-70{ }^{\circ} \mathrm{C}\right)$ por la pérdida del $60 \%$ del peso total de la muestra $(9.894 \mathrm{mg})$ debido a la liberación del agua fissisorbida en la secreción. Liberada el agua, la pérdida de peso en la secreción tiende a estabilizarse abarcado un rango de temperatura de los 90 a los $280{ }^{\circ} \mathrm{C}$ (Fig. 9) si se comparan estos dos eventos iniciales del comportamiento térmico de la secreción en estado líquido con la historia térmica inicial de la secreción adhesiva en estado sólido (Fig. 10), es posible inferir que la secreción en estado sólido al perder solo el $13 \%$ del peso total de la muestra $(0.190 \mathrm{mg})$ alcanza la estabilidad térmica a una temperatura más temprana $\left(100{ }^{\circ} \mathrm{C}\right)$ en comparación con la muestra líquida (Cuadro 5) y que el comportamiento del peso en la muestra de secreción sólida después de la fase de pérdida de agua a lo largo de todo el calentamiento es más estable que la muestra líquida. Los siguientes eventos térmicos del comportamiento de la secreción en 
CUADRO 5

Análisis termogravimétricos de la seda de diferentes especies de arañas y la secreción adhesiva de M. geagy

TABLE 5

Thermogravimetric analysis of silk from different species spiders and secretion adhesive of $\mathrm{M}$ geagy

\begin{tabular}{|c|c|c|c|c|}
\hline \multirow[b]{2}{*}{$\begin{array}{l}\text { Especie productora de seda } \\
\text { y secreción adhesiva }\end{array}$} & \multicolumn{2}{|c|}{ Evento térmico } & \multicolumn{2}{|c|}{ Desnaturalización } \\
\hline & $\begin{array}{l}\text { Perdida de peso } \\
\text { (pérdida de agua) }\end{array}$ & $\begin{array}{c}\text { Rango de } \\
\text { estabilización } \\
\text { térmica }\end{array}$ & $\begin{array}{c}\text { Rompimiento } \\
\text { cadenas laterales } \\
\text { de aminoácidos }\end{array}$ & $\begin{array}{c}\text { Degradación } \\
\text { cadena principal } \\
\text { de aminoácidos }\end{array}$ \\
\hline $\begin{array}{l}\text { Nephila (Araneidae) } \\
\text { (Seenivasan et al. 2005) }\end{array}$ & $\begin{array}{c}<150{ }^{\circ} \mathrm{C} \text { pérdida del } 7 \% \\
\text { del peso }\end{array}$ & $150-240 \mathrm{oC}$ & $240-500{ }^{\circ} \mathrm{C}$ & $500-890{ }^{\circ} \mathrm{C}$ \\
\hline $\begin{array}{l}\text { Plexippus (Salticidae) } \\
\text { (Jerez y Bernal 2007)* }\end{array}$ & $\begin{array}{c}<50{ }^{\circ} \mathrm{C} \text { pérdida del } 10 \% \\
\text { del peso }\end{array}$ & $60-100^{\circ} \mathrm{C}$ & $100-280{ }^{\circ} \mathrm{C}$ & $315-500{ }^{\circ} \mathrm{C}$ \\
\hline $\begin{array}{l}\text { Secreción adhesiva de } \\
\text { M. geagy (muestra líquida) }\end{array}$ & $\begin{array}{c}70{ }^{\circ} \mathrm{C} \text { pérdida del } 60 \% \\
\text { del peso }\end{array}$ & $90-280^{\circ} \mathrm{C}$ & $280-355^{\circ} \mathrm{C}$ & $355-485^{\circ} \mathrm{C}$ \\
\hline $\begin{array}{l}\text { Secreción adhesiva de } \\
\text { M. geagy (muestra sólida) }\end{array}$ & $\begin{array}{c}90{ }^{\circ} \mathrm{C} \text { pérdida del } 13 \% \\
\text { del peso }\end{array}$ & $100-205^{\circ} \mathrm{C}$ & $220-350{ }^{\circ} \mathrm{C}$ & $355-495{ }^{\circ} \mathrm{C}$ \\
\hline
\end{tabular}

*El manuscrito se encuentra en evaluación para publicación en la Revista Colombiana de Entomología.

estados sólido y líquido corresponden a la desnaturalización de la proteína en la muestra. La primera etapa del proceso de desnaturalización, atribuida al rompimiento de las cadenas laterales de los aminoácidos en la muestra líquida se observó entre los 280 y $355^{\circ} \mathrm{C}$ y entre los 220 y $350{ }^{\circ} \mathrm{C}$ en la muestra sólida. En esta fase también se observaron las diferencias en la pérdida de peso en cada una de las muestras. En la muestra líquida al terminar la primera fase de la desnaturalización se ha perdido cerca del $88 \%$, y en la muestra sólida el $65 \%$, a pesar de que el peso inicial de la muestra sólida fue menor que el de la muestra líquida. La última etapa de calentamiento en los termogramas la fase de descomposición, es atribuida a la degradación de la cadena principal de aminoácidos; en la muestra líquida esta fase se presentó en el rango de los 355 y $485{ }^{\circ} \mathrm{C}$ y entre los 355 y $495{ }^{\circ} \mathrm{C}$ en la muestra de secreción en estado sólido (Ver Cuadro 5). De acuerdo con el comportamiento térmico exhibido por la secreción adhesiva de $M$. geagy en estado líquido y sólido, y teniendo en cuenta el porcentaje de agua en la secreción adhesiva de los onicóforos ( $~ 90$ \%) (Read 1985, Roper 1977), es posible concluir que una vez disipada el agua presente en la muestra, esta es térmicamente estable dentro del rango de 100 y $300{ }^{\circ} \mathrm{C}$.

Este tipo de análisis termogravimétrico implementado en el estudio de la secreción adhesiva de $M$. geagy ha sido ampliamente utilizado en la caracterización térmica de las fibras de la seda producida por las arañas. Seenivasan y colaboradores en el 2005 caracterizaron mediante análisis de termogravimetría la seda producida por la araña Nephila (Tetragnathidae) catalogada como una de las más resistentes, ligeras y elásticas (Rossbach et al. 2003). Reportaron en la primera etapa del análisis la pérdida del $7 \%$ peso de la muestra atribuyéndolo a la pérdida de agua. Finalmente la estabilidad térmica para la fibra se reportó dentro del rango de los 150 y $240{ }^{\circ} \mathrm{C}$. En comparación con estos eventos térmicos característicos de la seda de la araña Nephila, la secreción adhesiva de $M$. geagy en estado sólido y líquido registró mayor pérdida en el porcentaje del 
peso 13 y $60 \%$ respectivamente a temperaturas más tempranas $\left(<90{ }^{\circ} \mathrm{C}\right)$, abarcando un rango de estabilidad térmica más amplio (100-280 ${ }^{\circ} \mathrm{C}$ ) alcanzado a temperaturas menores que las reportadas para la seda de la araña. Cerca de los $240{ }^{\circ} \mathrm{C}$ fue reportado el inicio de la fase de desnaturalización de la proteína en la fibra de la seda de araña (Seenivasan et al. 2005). La secreción adhesiva en estado sólido de M. geagy inicia la fase de desnaturalización a los $220^{\circ} \mathrm{C}$ (ver Cuadro 5). De acuerdo con lo anterior la secreción adhesiva de $M$. geagy después de la disipación del contenido de agua exhibe un comportamiento térmicamente estable a temperaturas más tempranas pero menos prolongado iniciando la fase de degradación más prontamente que la fibra de la seda de la araña Nephila.

En un reporte preliminar estudiamos el comportamiento térmico mediante termogravimetría de la seda del refugio de Plexxipus paykulli (Salticidae) y calculamos el porcentaje de láminas beta $(\beta)$ en la proteína de la seda del refugio mediante espectroscopía de infrarrojos. En el análisis termogravimétrico la pérdida inicial de peso en la muestra se observó entre los 20 y $50{ }^{\circ} \mathrm{C}$ perdiéndose el $10 \%$ del peso inicial $(0.041 \mathrm{mg})$. Este evento es similar al observado en la secreción adhesiva en estado sólido de M. geagy en la que se pierde el $8 \%$ del peso entre los primeros 40 y $50{ }^{\circ} \mathrm{C}$ del análisis. La fase de estabilización en la seda del refugio de la araña inicia a temperaturas más tempranas $\left(60{ }^{\circ} \mathrm{C}\right)$ y dentro de un rango de temperatura más corto en comparación con la secreción adhesiva sólida $\left(100^{\circ} \mathrm{C}\right)$ y líquida $\left(90^{\circ} \mathrm{C}\right) \mathrm{de}$ $M$. geagy, lo que conlleva a una temperatura de inicio de la degradación igualmente más temprana (ver Cuadro 5). La similitud entre el comportamiento térmico inicial de la seda del refugio de la araña saltarina y el de la secreción adhesiva sólida de $M$. geagy podría atribuirse a un reducido porcentaje de agua en las muestras. En las fibras de la seda producidas por las arañas porcentajes significativos de agua presentes en la muestra son asociados con fibras de redes tejidas para depredación (Saravanan, 2006). Plexippus paykulli de acuerdo con su habilidad para alcanzar grandes distancias y saltar sobre la presa, está asociada con la ausencia de tejido de redes para captura, sin embargo, teje refugios con fibras menos flexibles pero más rígidas como mecanismo de defensa ante los posibles depredadores (Flores 1996).

DSC vs TG de la muestra líquida: De acuerdo con los análisis de calorimetría diferencial, el flujo de calor en la secreción líquida de $M$. geagy se caracterizó en un evento endotérmico que abarcó un área de flujo de calor de $-1078 \mathrm{~J} / \mathrm{g}$ entre los 26 y $93{ }^{\circ} \mathrm{C}$ (ver Cuadro 3 y Fig. 11), esta energía absorbida estaría asociada con la ruptura de puentes de hidrógeno y la disipación de agua en la muestra corroborado la pérdida inicial de peso registrada en el análisis termogravimétrico de la secreción adhesiva liquida y sólida (Fig. 9 y 10). Posterior a liberación de agua, el flujo de calor en la muestra tiende a estabilizarse (Fig. 11) en similitud con la fase de estabilización de la pérdida de peso en el análisis termogravimétrico $\left(100-280{ }^{\circ} \mathrm{C}\right)$ (Fig. 9).

DSC vs TG de la muestra sólida: El flujo de calor en este tipo de muestra inicialmente se registró con un evento endotérmico que abarcó un área de flujo de calor de -794.6 $\mathrm{J} / \mathrm{g}$ desde los 27 hasta los $119{ }^{\circ} \mathrm{C}$. Dentro de este rango de temperatura en el termograma del análisis termogravimétrico se observa la pérdida de peso en la muestra y el inicio de la estabilización térmica de la muestra. Posterior a este primer evento de absorción de energía se observó una fase de agregación molecular en la que el flujo de calor exhibió una leve estabilización manteniéndose en $-7.469 \mathrm{~W} / \mathrm{g}$ entre los 115 y $175^{\circ} \mathrm{C}$ (Fig. 12), este evento se ajusta en el análisis termogravimétrico con la estabilización de la pérdida de peso en la muestra entre los 100 y $205^{\circ} \mathrm{C}$. El siguiente suceso de flujo de calor registrado como la fase inicial de la etapa de desnaturalización, el rompimiento de las cadenas laterales de los aminoácidos, se registró en un pico exotérmico entre los 242 y $269{ }^{\circ} \mathrm{C}$ abarcando un área de flujo de calor de $132.2 \mathrm{~J} / \mathrm{g}$. En el análisis termogravimétrico 
dentro de este rango de temperatura también es registrada la fase de desnaturalización. El evento endotérmico observado en la última etapa del calentamiento es atribuido a la degradación de la cadena principal de los aminoácidos, observándose una estabilización del flujo de calor entre los 315 y $383{ }^{\circ} \mathrm{C}$. En el análisis termogravimétrico este rango de temperatura igualmente abarca la etapa de desnaturalización de la muestra.

El porcentaje de laminas $\beta$ calculado dentro de la región de la banda amida I del espectro infrarrojo de la secreción adhesiva de $M$. geagy fue de $59 \%$. Este tipo de conformación estructural ha sido asociado con las características mecánicas exhibidas por las fibroinas de la seda producidas por insectos y arañas como la de la seda producida por la glándula ampollácea de Nephila (Stubbs et al. 1992), asimismo cerca del $100 \%$ de la proteína en la fibra de la seda del capullo de Bombix mori el gusano de la seda, ha presentado conformación estructural en laminas $\beta$ (Lucas et al. 1960).

El porcentaje de laminas $\beta$ calculado en la seda del refugio de Plexippus paykulli fue de $67 \%$. El cálculo del porcentaje de láminas $\beta$ en la muestra de seda producida por esta araña nos permitió hacer la comparación con la secreción de los onicóforos ya que este tipo de análisis se reportan únicamente para fibras pre-tratadas con solventes químicos y no en su estado nativo (Gandhi 2006). La cantidad de proteína expuesta para cada uno de los tres motivos estructurales (helice $\alpha$, hoja $\beta$ y giros $\beta$ ) afecta el comportamiento mecánico de las fibras y por lo tanto es un valor potencial selectivo de los organismos que las producen (Simmons et al. 1996), estas conformaciones de tipo estructural confieren cierta complejidad mecánica a las fibras (Craig et al.1994). Lo que quiere decir que, la estructura en láminas $\beta$ presentes en la secreción adhesiva de los onicóforos al igual que en las fibras de seda de las arañas le confiere extensibilidad y resistencia a la tracción, características en la fibra de la seda de Nephila demandan mayor energía para romper el mismo volumen de proteína de la seda que el de cualquier otro material biológico (Denny 1980, Gosline et al. 1984). Ruhberg y Storch en 1977 reportaron estas propiedades de extensibilidad y resistencia a la tracción de los hilos formados por la secreción adhesiva de los onicóforos al ser expulsada desde las papilas orales. El componente adhesivo en la secreción de los onicóforos se distribuye en gotas viscosas espaciadas que se observan distribuidas a lo largo de los hilos (Benkendorff et al. 1999) en similitud con las fibras de seda de las arañas tejidas para depredación (Vollrath y Tillinghast 1991). Las propiedades adhesivas de la secreción estarían atribuidas a la viscosidad de la misma; cuando el volumen de la secreción es grande y es expulsado desde las papilas orales sobre la superficie de la presa, la secreción llena los espacios entre la superficie de la secreción y la superficie de la presa formados cuando ésta trata de huir evitando que la brecha entre el fluido y la superficie de la presa se amplíe, logrando la adhesión total de la secreción a la superficie de la presa.

La composición de aminoácidos rica en glicina y prolina de la secreción adhesiva de los onicóforos sugieren la presencia de un material tipo colágeno (Benkendorff et al. 1999). De acuerdo con lo anterior la secreción adhesiva de los onicóforos ha sido asociada con la modalidad de estructura secundaria de tipo, hélice de colágeno en la que el aminoácido glicina es el único que puede encajar en el interior de la hélice (Craig et al. 1994). Sin embargo la frecuencia de longitud de onda atribuida al tipo de conformación hélice $\alpha$ en el espectro infrarrojo de la secreción de $M$. geagy fue baja. Giros- $\beta$ fueron identificados en el espectro infrarrojo de la secreción adhesiva, este tipo de conformación en la banda amida I se observó en 1685 longitudes de onda (Fig. 14). Las sedas de colágeno de los artrópodos son de tipo giros- $\beta$ y son producidas por una glándula dedicada exclusivamente a la producción de seda (Sehnal y Akai 1990), como las papilas orales de los onicóforos y las glándulas salivales de la avispa portasierra Nematus ribesii.

La secreción adhesiva de los onicóforos de acuerdo con los análisis termogramétricos, 
calorimétricos y de espectroscopía de infrarrojos se caracteriza por ser un biomaterial térmicamente estable en el rango de los 90 y $280{ }^{\circ} \mathrm{C}$ en estado líquido y en el rango de los 100 y $205{ }^{\circ} \mathrm{C}$ en estado sólido sufriendo pérdidas de peso en las primeras etapas del calentamiento debido a la liberación de agua fisisorbida en la muestra. De acuerdo con la caracterización de los componentes estructurales de la estructura secundaria de la proteína en la secreción adhesiva, el $59 \%$ es atribuido a la conformación de tipo estructural lámina $\beta$. Con la implementación de este tipo de análisis de termogravimetría, calorimetría y espectroscopía de infrarrojos en el estudio de la secreción adhesiva de $M$. geagy complementados con análisis moleculares, bioquímicos y físicomecánicos, es posible determinar la utilidad de la secreción adhesiva de los onicóforos en futuras investigaciones que busquen nuevos materiales de origen natural para la fabricación de nuevos pegamentos o biosuturas, fibras de origen natural útiles en la industria textil y en la medicina ofreciendo nuevas alternativas para la elaboración de materiales adhesivos.

\section{AGRADECIMIENTOS}

Agradecemos a Guillermo Acero Medina del Laboratorio de Análisis térmico de la Escuela de Ingeniería Química de la Universidad Industrial de Santander por la asesoría en el tratamiento de las muestras de la secreción adhesiva de los onicóforos en los análisis térmicos. Para la captura de los onicóforos en el área estudiada se obtuvo el debido permiso de la Corporación Autónoma Regional de Santander (CAS) y la Corporación para la Defensa de la Meseta de Bucaramanga (CDMB).

\section{RESUMEN}

Redescribimos taxonómicamente el gusano Macroperipatus geagy Bouvier 1899, a partir de 15 especímenes recolectados en los cafetales de hacienda El Roble, Santander, Colombia. De acuerdo con la reconstrucción en tres dimensiones de la superficie dorsal del cuerpo, las papilas primarias y accesorias de $M$. geagy se distribuyen sin formar un patrón determinado. Características diagnósticas: un diente accesorio y un diente principal en la mandíbula externa; y un diente principal, un diente accesorio y siete dentículos en la sierra de la mandíbula interna. Cuerpo: longitud $(X=45.66 ; S=26.10 \mathrm{~mm})$, peso $(X=0.95$; $\mathrm{S}=2.21)$ y pares de lobopodos $(\mathrm{X}=28.13 ; \mathrm{S}=1.30)$. Se elaboró una clave para seis especies de Macroperipatus. La secreción adhesiva en estado líquido perdió $60 \%$ del peso inicial al alcanzar $\operatorname{los} 70^{\circ} \mathrm{C}$, la muestra sólida perdió el $13 \%$ del peso cerca de $\operatorname{los} 90{ }^{\circ} \mathrm{C}$, la fase de estabilidad térmica de la secreción estuvo entre los 90 y $280^{\circ} \mathrm{C}$ en la secreción líquida y entre los 100 y $205{ }^{\circ} \mathrm{C}$ en la sólida. La temperatura de inicio de la degradación de las muestras se registró a $\operatorname{los} 355{ }^{\circ} \mathrm{C}$. En comparación con la araña Nephila sp., el onicóforo perdió mayor porcentaje de peso y alcanzó la fase de degradación a temperaturas más bajas. Los cambios en el flujo de calor en la secreción adhesiva sólida y líquida de $M$. geagy fueron confirmados con el comportamiento de las muestras en el análisis de termogravimetría. La proporción de láminas beta calculado en el espectro infrarrojo de la secreción adhesiva fue del 59\%. La presencia de este tipo de conformación de la estructura secundaria de las proteínas en la secreción adhesiva de $M$. geagy conferiría extensibilidad y resistencia a la tracción, en similitud con las fibras de seda tejidas por las arañas para depredación.

Palabras clave: gusanos aterciopelados, Macroperipatus geagy, dentículos, mandíbula interna, hoja beta, espectro infrarrojo, seda de arañas.

\section{REFERENCIAS}

Benkendorff, K., K. Beardmore, A. Gooley \& N. Parker. 1999. Characterisation of the slime gland secretion from the peripatus, Euperipatus kanangrensis (Onychophora: Peripatopsidae). Comp. Biochem. Physiol. 124: 457- 465.

Bouvier, E. L. 1907. Monographie des Onychophores. II. Annales des Sciences Naturelles, Zoologie et Biologie Animale 9e Série 5: 61-318.

Bouvier, E. L. 1905. Monographie des Onychophores. I. Annales des Sciences Naturelles, Zoologie et Biologie Animale 9e Série 2: 1-383.

Bouvier, E. L. 1899b. Contributions a l'histoire des Peripates Americains. Ann. Soc. Ent. Fr. 68: 386450.

Brues, C. T. 1941. Peripatus (Macroperipatus) geagy in Panama. Phyche 48: 111-112.

Brues, C. T. 1925. Notes on Neoptropical Onychophora. Psyche 32: 159-165.

Chirgadze, Y., O. Fedorov \& T. Trushina. 1975. Estimation of amino acid residue side-chain absorption in the 
infrared spectra of protein solution in heavy water. Biopolymers 14: 679-694.

Craig, C., G. Bernard \& J.A. Coddington. 1994. Evolutionary shifts in the spectral properties of spider silks. Evolution 48: 287-96.

Clark, A. 1913. A revision of the American species of Peripatus. Proc. Biol. Soc. Wash. 26: 15-20.

Denny, M. 1980. Silks-their properties and functions. In J.F.V. Vincent \& J.D. Currey Soc. (eds.). The Mechanical Properties of biological materials Exp. Biol. 247-71.

Dong, A. \& W. Caughey. 1990. Protein secondary structure in water from second-derivative amide I infrared spectra. Biochemistry 29: 3303-3308.

Evans, R. 1901a. On two new species of Onychophora from the Siamese Malay States. Q. J. Microsc. Sci. $45: 473-538$

Flores, E. 1996. Las arañas del Departamento del Valle del Cauca. Un manual introductorio a su diversidad y clasificación. Cali (Colombia): Inciva y Colciencias, Universidad del Valle, Colombia.

Gandhi, M. R. 2006. Silk protein as a biomaterial for tissue engineering application: Theoretical and experimental study. Ph.D. Thesis, Drexel University, Filadelfia, EEUU.

Gleeson, D. 1996. Onychophora of New Zealand; past, present and future. New Zealand Entomologist 19: 51-55.

Gosline, J., M. Demont \& M. Denny. 1984. The structure and properties of spider silks. Endeavour 10: 37-43.

Hayashi, C. \& R. V. Lewis. 1998. Evidence from flagelliform silk cDNA for the structural basis of elasticity and modular nature of spider silks. Journal of Molecular Biology 275: 773-784.

Johnson, W., L. Delanney, E. Williams \& T. Cole. 1969. Principles of Zoology. Holt, Rinehart and Winston Inc., Nueva York, EEUU.

Lavallard, R. \& S. Campiglia. 1975. Contribution á la biologie de Peripatus acaioi Marcus and Marcus (Onychophore). V. Etude des naissances dans un élevage de laboratoire. Zool. Anz. 195: 338-350.

Lucas, F., J. Shaw \& S. Smith. 1960. Comparative studies of fibroins. J. Mol. Biol. 2: 339-349.

Mayer, G. 2007. Metaperipatus inae sp. nov. (Onychophora: Peripatopsidae) from Chile with a novel ovarian type and dermal insemination. Zootaxa 1440: 21-37.
Mcglynn, T. \& C. Kelley. 1999. Distribution of a Costa Rican wet forest velvet worm (Onychophora: Peripatidae). Ecology and Population Biology 2: 53-55.

Monge-Nájera, J., Z. Barrientos \& F. Aguilar. 1993. Behavior of Epiperipatus biolleyi (Onychophora: Peripatidae) under laboratoty conditions. Rev. Biol.. Trop. 41: 689-696.

Monge-Nájera, J. \& B. Morera. 1994. Morphological and physiological characteristics of two species of Epiperipatus from Costa Rica (Onychophora: Peripatidae). Rev. Biol. Trop. 42: 181-188.

Monge-Nájera, J. 1994a. Ecological Biogeography in the Phylum Onychophora. Biogeographica 70: 111-123.

Monge-Nájera, J. 1994b. Reproductive trends, habitat type and body characteristics in velvet worms (Onychophora). Rev. Biol. Trop. 42: 611-622.

Monge-Nájera, J. 1995. Phylogeny, biogeography and reproductive trends in the Onychophora. Zool. J. Linn. Soc. 114: 21-60.

Monge-Nájera, J. \& J. P. Alfaro. 1995. Geographic variation of habitats in Costa Rican velvet worms (Onychophora: Peripatidae). Biogeographica 71: 97-108.

Monge-Nájera, J., Z. Barrientos \& F. Aguilar. 1996. Experimental behaviour of tropical invertebrate: Epiperipatus biolleyi (Onychophora: Peripatidae). Mem. Mus. Nac. Hist. Nat. 169: 434-439.

Monge-Nájera, J. \& X. Hou. 1999. 500 Millones de años de evolución: onicóforos, los primeros animales que caminaron (Onychophora). Boletín S.E.A. 26: 171-178.

Morera-Brenes, B. \& M. D. León. 1986. Description of Macroperipatus valerioi n. sp. from Costa Rica, and comments on the genus Macroperipatus. (Onychophora: Peripatidae). Rev. Biol. Trop. 34: 277-282.

Morera-Brenes, B., J. Monge-Nájera \& R. Sáenz. 1988. Partiturition in Onychophorans: new record and a review. Brenesia 29: 15-20.

Morera-Brenes, B. \& J. Monge-Najera. 1990. Ephiperipatus hilkae, n. sp. from Costa Rica (Onychophora: Peripatidae). Rev. Biol.. Trop. 38: 449-455.

Oliveira, I. S. \& A. H. Wieloch. 2005. Macroperipatus machadoi sp. n. (Onychophora: peripatidae) da Floresta Atlántica de Minas Gerais, Brasil. Lundiana 6: 61-66. 
Øyvind Hammer, David A.T. Harper \& P.D. Ryan. 2005 Palaeontological Statistics, ver. 1.81.

Peck, S. B. 1975. A review of the new world Onychophora with the description of a new cavernicolous genus and species from Jamaica. Psyche 82: 341-358.

Peraza, C., Y. Cifuentes, Y. Alayon \& C. Clavijo. 2004. Adiciones a la avifauna de un cafetal con sombrío en la Mesa de Los Santos (Santander, Colombia). Universitas Scientiarum. Revista de la Facultad de Ciencias. Pontificia Universidad Javeriana 9:19-32.

Read, V.M. 1985. The ecology of Macroperipatus torquatus (Kennel) with special reference to feeling and taxonomic review. Ph.D. Thesis, Univerity College of North Wales, Bangor, Reino Unido.

Read, V.M. St. J. 1986. Clave para identificación de los Onicóforos del Ecuador. Publicaciones Museo Ecuatoriano de Ciencias Naturales, Ecuador.

Read, V. M. \& R. N. Hughes. 1987. Feeding behavior and prey choice in Macroperipatus torquatus (Onychophora). The Royal Society 230: 483-506.

Roper, H. 1977. Analytical Investigations of defensive secretions from Peripatopsis moseleyi (Onychophora). Nature 32: 56-60.

Rossbach, V., P. Patanathabutr \& J. Wichitwechkarn. 2003. Copying and manipulating nature: innovation for textile materials. Fibres and polymers 4: 8-14.

Ruhberg, H. 1985. Die Peripatopsidae (Onychophora). Systematik, Ökologie, Chorologie

and phylogenetische aspekte. Zoologica 137: 1-183.

Ruhberg, H. \& V. Storch. 1977. U8 ber Wehrdru“sen und Wehrsekret von Peripatopsis moseleyi (Onychophora). Zool. Anz. 198: 9-19.

Rupert, E. E. \& Barnes, R. D. 1996. Zoología de Invertebrados. McGraw-Hill Interamericana. México.
Saravanan, D. 2006. Spider silk-structure, properties and spinning. Journal of Textile and Apparel Technology and Management 5: 1-20.

Seenivasan, R., M. Jassal \& C. Rameshkumar. 2005. Studies on structure and properties of Nephila-spider silk dragline. Autex Research Journal 5: 30-39.

Sehnal, F. \& H. Akai. 1990. Insect silk glands: their types, development and function, and effects of environmental factors and morphogenetic hormones on them. Int. J. Insect. Morphol. Embryol.19: 79-132.

Simmons, A. H., C.A. Michal \& L.W. Jelinski. 1996. Molecular orientation and two component nature of the crystalline fraction of spider dragline silks. Science 271: 84-87.

Storch, V. \& H. Ruhberg. 1993. Onychophora, p. 11-56. In Harrison, F.W. \& M.E Rice (eds.) Microscopic Anatomy of Invertebrates. Vol. 12. Wiley-Liss, Nueva York, EEUU.

Stubbs, D.G., E.K. Tillinghast \& M.A. Townley. 1992. Fibrous composite structure in a spider silk. Naturwissenschaften 79: 231-234.

Strausfeld, N. J., C. M. Strausfeld, R. Loesel, D. Rowell \& S. Stowe. 2006. Arthropod phylogeny: onychophoran brain organization suggests an archaic relationship with a chelicerate stem lineage. Proc. Roy. Soc. 273: 1857-1866.

Vasconcellos, A., Almeida, W. \& Souza, L. 2006. Onychophora in humid forests of northeastern Brazil. Braz. J. Biol. 66: 187-189.

Venyaminov, S.Y. \& N.N. Kalnin. 1990. Quantitative IR spectrophotometry of peptide compounds in water $\left(\mathrm{H}_{2} \mathrm{O}\right)$ solution. II. Amide absorption bands of polypeptide and fibrous protein in $\alpha-, \beta$ - and random coil conformations. Biopolymers 30: 1251-1279.

Vollrath,F.\& E. Tillinghast. 1991. Glycoprotein glue beneath a spider web's aqueous coat. Naturwissenschaften 78 : 557-559. 